\title{
Development and validation of a collagen signature-based nomogram for preoperatively predicting lymph node metastasis and prognosis in colorectal cancer
}

\author{
Meiting Fu ${ }^{1 \#}$, Dexin Chen ${ }^{2 \#}$, Fuzheng Luo ${ }^{1 \#}$, Guangxing Wang ${ }^{3,4}$, Shuoyu $\mathrm{Xu}^{2}$, Yadong Wang ${ }^{1}$, \\ Caihong Sun ${ }^{3,4}$, Xueqin $\mathrm{Xu}^{3,4}$, Aimin $\mathrm{Li}^{1}$, Shuangmu Zhuo ${ }^{3,4}$, Side Liu ${ }^{1}$, Jun Yan ${ }^{2}$ \\ ${ }^{1}$ Department of Gastroenterology, Nanfang Hospital, Southern Medical University, Guangzhou, China; ${ }^{2}$ Department of General Surgery, Nanfang \\ Hospital, Southern Medical University, Guangzhou, China; ${ }^{3}$ School of Science, Jimei University, Xiamen, China; ${ }^{4}$ Key Laboratory of OptoElectronic \\ Science and Technology for Medicine of Ministry of Education, Fujian Normal University, Fuzhou, China \\ Contributions: (I) Conception and design: A Li, S Zhuo, S Liu, J Yan; (II) Administrative support: A Li, S Liu, J Yan; (III) Provision of study materials \\ or patients: M Fu, D Chen, F Luo, A Li, S Liu, J Yan; (IV) Collection and assembly of data: M Fu, D Chen, F Luo, G Wang, S Xu, Y Wang, C Sun, \\ $\mathrm{X} \mathrm{Xu}$; (V) Data analysis and interpretation: M Fu, D Chen, F Luo, J Yan; (VI) Manuscript writing: All authors; (VII) Final approval of manuscript: \\ All authors. \\ \#These authors contributed equally to this work. \\ Correspondence to: Jun Yan. Department of General Surgery, Nanfang Hospital, Southern Medical University, Guangzhou, China. Email: \\ yanjunfudan@163.com; Side Liu. Department of Gastroenterology, Nanfang Hospital, Southern Medical University, Guangzhou, China. Email: \\ liuside2011@163.com; Shuangmu Zhuo. School of Science, Jimei University, Xiamen, China. Email: shuangmuzhuo@gmail.com; Aimin Li. \\ Department of Gastroenterology, Nanfang Hospital, Southern Medical University, Guangzhou, China. Email: lam0725@163.com.
}

Background: Current preoperative evaluation approaches cannot provide adequate information for the prediction of lymph node (LN) metastasis in colorectal cancer (CRC). Collagen alterations in the tumor microenvironment affect the progression of tumor cells. To more accurately assess the LN status of CRC preoperatively, we developed and validated a collagen signature-based nomogram for predicting LN metastasis in CRC.

Methods: In total, 342 consecutive CRC patients were assigned to the training and validation cohorts. A total of 148 fully quantitative collagen features were extracted based on preoperative biopsies using multiphoton imaging, and the least absolute shrinkage and selection operator method was utilized to construct the collagen signature. A collagen signature-based nomogram was developed by multivariable logistic regression in the training cohort. Nomogram performance was evaluated for its discrimination, calibration, and clinical usefulness and then validated in the validation cohort. The prognostic values of the nomogram were also evaluated.

Results: A seven-feature-based collagen signature was built. We found that the collagen signature showed a significant association with LN metastasis in CRC. Additionally, a nomogram incorporating preoperative tumor differentiation, computed tomography-reported T stage and LN status, carcinoembryonic antigen level, carbohydrate antigen 19-9 level and collagen signature was developed. This nomogram had good discrimination and calibration, with AUROCs of 0.826 and 0.846 in the training and validation cohorts, respectively, and had a sensitivity of $86.5 \%$, a specificity of $68.2 \%$, an accuracy of $76.9 \%$, a negative predictive value of $84.9 \%$, and a positive predictive value of $71.2 \%$ for all patients. Compared to the clinicopathological model, which consisted of the clinicopathological risk factors for LN metastasis, the collagen signature-based nomogram demonstrated a significantly improved ability to discriminate LN status. Moreover, a nomogram-predicted high-risk subgroup had remarkably reduced survival compared with that of the low-risk subgroup.

Conclusions: The collagen signature in the tumor microenvironment of preoperative biopsies is an independent predictor for LN metastasis in CRC, and the collagen signature-based nomogram is helpful for tailored treatment and prognostic predictions in CRC preoperatively. 
Keywords: Colorectal cancer (CRC); lymph node metastasis; collagen signature; nomogram; prognosis

Submitted Nov 19, 2020. Accepted for publication Jan 22, 2021.

doi: $10.21037 / \mathrm{atm}-20-7565$

View this article at: http://dx.doi.org/10.21037/atm-20-7565

\section{Introduction}

Colorectal cancer (CRC), a major malignancy, represents the second deadliest cancer worldwide (1). Accurate assessment of lymph node (LN) metastasis is important for treatment decisions and prognostic predictions for CRC patients (2). Preoperative evaluation of LN status can help determine the need for adjuvant/neoadjuvant therapy and the adequacy of surgical resection, thereby aiding in pretreatment decision-making. Some histopathologic parameters, such as lymphovascular infiltration, are predictive of $\mathrm{LN}$ metastasis in CRC but are available only postoperatively (3). Several imaging methods, including computed tomography (CT), are limited in terms of detecting actual high-risk patients with $\mathrm{LN}$ metastasis, resulting in remarkable case understaging or overstaging (4). Therefore, a more robust biomarker is urgently required to improve the accuracy of nodal staging preoperatively.

The extracellular matrix (ECM) is a highly specialized scaffold through which cancer cells reside in tissues, and the interaction between cancer cells and the ECM regulates diverse cellular functions, such as growth, differentiation and migration (5). As the main component of the ECM, collagen is responsible for most ECM functions in the tumor microenvironment $(6,7)$. Collagen reorganization in the tumor microenvironment has been shown to be a metastatic and prognostic biomarker in several solid tumors (8-10). In the clinic, preoperative biopsies obtained from colonoscopic examination are adequate for determining the malignancy status of CRC; thus, it would be helpful to acquire a predictive biopsy-based biomarker to improve the accuracy of nodal staging. However, the association between collagen alterations in preoperative biopsies of CRC and LN metastasis has not yet been investigated. Herein, we hypothesize that collagen alterations in the tumor microenvironment of the preoperative biopsy are associated with $\mathrm{LN}$ metastasis in patients with CRC.

As an imaging modality involving the combination of two-photon excitation fluorescence (TPEF) with second harmonic generation (SHG), multiphoton imaging has been increasingly applied in the field of biological medicine (11).
Because of its underlying physical properties, multiphoton imaging has emerged as a powerful modality for collagen imaging in a broad range of tissues (12). The SHG signals of multiphoton imaging are highly sensitive to collagen structure and, importantly, to changes that occur in the tumor microenvironment (13). In addition, collagen features, including morphological and textural features, can be extracted from multiphoton images to comprehensively describe collagen alterations $(14,15)$.

Integrating multiple factors into a single biomarker would yield more powerful and accurate prediction performance $(16,17)$. The least absolute shrinkage and selection operator (LASSO) method is a popular method for variable selection with high-dimensional data $(18,19)$. Here, we propose a collagen signature that is derived from multiple LN metastasis-associated collagen features of preoperative biopsies based on multiphoton imaging and the LASSO regression method. Therefore, in this study, we aimed to develop and validate a nomogram that incorporated the collagen signature from biopsies and clinicopathological risk factors for individual preoperative prediction of LN status in CRC. In addition, we evaluated the prognostic value of the nomogram.

We present the following article in accordance with the TRIPOD reporting checklist (available at http://dx.doi. org/10.21037/atm-20-7565).

\section{Methods}

This retrospective study was approved by the institutional review board of Nanfang Hospital, Southern Medical University, which waived the requirement to obtain written informed consent and was carried out according to the Declaration of Helsinki (as revised in 2013) for biomedical research involving human subjects.

\section{Study design and participants}

The present study was conducted in CRC patients treated between October 1, 2015, and June 30, 2018, at 
the Nanfang Hospital of Southern Medical University (Guangzhou, China). The inclusion criteria were as follows: (I) pathologically confirmed stage I-III CRC; (II) surgical resection with curative intent; (III) lymphadenectomy performed with at least $12 \mathrm{LNs}$ harvested; (IV) complete clinicopathological and follow-up data; and (V) availability of preoperative formalin-fixed and paraffin-embedded (FFEP) biopsies. Individuals with double or multiple primary tumors or receiving neoadjuvant anticancer therapy were excluded (Figure S1). Finally, a total of 342 consecutive patients were enrolled for analysis. Subsequently, computergenerated random numbers were used to assign $70 \%$ and $30 \%$ of patients to the training and validation cohorts, respectively.

Patient clinicopathological information, including age, sex, tumor location; carcinoembryonic antigen (CEA) and cancer antigen 19-9 (CA 19-9) levels; CT-reported tumor size, T stage, and LN status; tumor differentiation and histological type from biopsies; histological type, tumor differentiation, $\mathrm{T}$ stage, and LN status from surgical specimens; and follow-up data (follow-up duration and survival status), was collected. The CEA and CA 19-9 levels, as well as CT-reported results, were obtained from routine preoperative examinations within one week before surgery. The primary outcome was the pathological diagnosis of LN metastasis after surgery.

\section{Acquisition of multiphoton images and selection of regions of interest}

Multiphoton imaging was performed on a section of each FFPE biopsy with a $20 \times$ objective using multiphoton microscopy. The multiphoton microscopy used in this study has been described previously (20). Briefly, the system contained a high-throughput scanning inverted Axiovert 200 microscope (LSM 510 META; Zeiss, Germany) and was equipped with a mode-locked femtosecond titanium (Ti): sapphire laser (110 fs, $76 \mathrm{MHz}$ ), tunable from 700 to $980 \mathrm{~nm}$ (Mira 900-F; Coherent, America). An acoustooptic modulator was used to control the attenuation of the laser intensity. A plan-apochromat 20x objective (Zeiss) was utilized for focusing the excitation beam and for collecting the backward signals. The META detector collected the backward multiphoton signals from the tissue sample. The two-channel mode could achieve TPEF and SHG signals, which were separated by the dichroic mirror in the detection path. One channel corresponds to a wavelength range of 430 to $708 \mathrm{~nm}$ to show the cell morphologies from the TPEF signals, whereas another channel covered the wavelength range from 390 to $410 \mathrm{~nm}$ to present the microstructures of collagen from the SHG signals. The excitation wavelength $\left(\lambda_{\mathrm{ex}}\right)$ was $810 \mathrm{~nm}$.

The 5- $\mu \mathrm{m}$ hematoxylin-eosin (H\&E)-stained slides of all enrolled patients were prepared on the other serial section. After acquisition of the multiphoton images of the biopsies, the corresponding $\mathrm{H} \& \mathrm{E}$-stained slides were used for histologic assessment by a pathologist who was blinded to the LN status of each patient, and three regions of interest within the tumor tissues (field of view, $500 \times 500 \mu \mathrm{m}$ ) were randomly selected for extraction of collagen features from multiphoton images.

\section{Extraction of collagen features}

The extraction of collagen features was performed using MATLAB 2015b (MathWorks) (14,15). For morphological features, the SHG image was first segmented into collagen pixels and background pixels using the Gaussian mixture model method (21). The binary collagen mask image was then processed using a fiber network extraction algorithm to trace each collagen fiber in the image and identify the cross-link points, which are defined as connecting points between two or more fibers (22). Moreover, we quantified an orientation index to reflect the collagen alignment based on Fourier transform spectra (23). Seven morphological features were extracted, namely, the collagen area, number, length, width, straightness, crosslink density and orientation, and the mean and variation in each morphological feature were calculated. For textural features, a histogram-based approach was first used. The mean, variation, skewness, kurtosis, energy and entropy were calculated from the histogram of the SHG pixel intensity distribution. Then, eighty gray-level cooccurrence matrix (GLCM)-based texture features were extracted (24). The contrast, correlation, energy and homogeneity were calculated from the GLCM with five different displacements of pixels at 1, 2, 3, 4 and 5 and four different directions at $0,45,90$ and 135 degrees. Furthermore, forty-eight Gabor wavelet transform features were also extracted for analysis (25). To calculate the Gabor wavelet transform features, the SHG images were convolved with Gabor filters at five different scales and six different orientations, and the mean and variation in the magnitude of the convolution over the image at each setting were calculated. Finally, a 
total of 148 features were extracted (Table S1).

\section{Construction of collagen signature}

The LASSO regression method applying a 10-fold crossvalidation was used for selecting a panel of the most predictive features in the training cohort, which was an accepted approach for the regression of high-dimensional data $(18,19)$. The method used an L1 penalty to shrink some regression coefficients to exactly zero. A penalty parameter $\lambda$ was selected via $1-\mathrm{SE}$ (standard error) criteria, namely, the optimal $\lambda$ was the largest value for which the area under the receiver operator characteristic (ROC) curve (AUROC) was within one SE of the largest value of the AUROC in the training cohort. Thereafter, a multiplefeature-based collagen signature was constructed based on a linear combination of the nonzero regression coefficients derived from the LASSO regression and the corresponding features. The collagen signature in the validation cohort was obtained directly, according to the formula used in the training cohort.

\section{Association of collagen signature with LN status}

The potential association between the collagen signature and LN status was first assessed in the training cohort and then validated in the validation cohort. Then, stratified analyses were carried out based on various subgroups. The discrimination of the collagen signature was evaluated using the AUROC.

\section{Development and evaluation of the nomogram}

All preoperative clinicopathological variables and the collagen signature were assessed by univariable analysis for their associations with $\mathrm{LN}$ metastasis in the training cohort, and variables showing $\mathrm{P}<0.05$ were selected for the multivariate analysis. A multivariate prediction model was constructed, and a nomogram was developed. The multicollinearity of the multivariate prediction model was assessed using the tolerance and variance inflation factor (26). Discrimination and calibration were used to evaluate the performance of the nomogram (27). For quantification of the discrimination of the nomogram, the AUROC was measured. The calibration of the nomogram was evaluated by the calibration curve to assess the goodness of fit, accompanied by the Hosmer-Lemeshow test (28).

\section{Validation of the nomogram}

The bootstrap method was used for internal validation, in which random samples drawn with replacement from the original data set were the same size as the training cohort (29). One thousand bootstrap repetitions were performed, and the mean concordance index was calculated. The nomogram was then applied in the validation cohort for external validation, with the AUROC calculated and calibration curve plotted.

\section{Clinical usefulness of the nomogram}

A decision curve analysis was performed to illustrate the clinical usefulness of the nomogram by calculating the net benefits at different threshold probabilities in both the training and validation cohorts (30). The context for decision curve analysis was a situation in which individuals' risks for an undesirable outcome were assessed, and individuals with sufficiently high risk were recommended for some intervention or treatment (31). The decision curve analysis provided a net benefit, which was calculated using the following formula:

Net benefit $=$ True positive rate - false positive rate $\times \frac{P_{t}}{1-P_{t}}[1]$

In the formula, $P_{\mathrm{t}}$ was the threshold probability where the expected benefit of treatment was equal to the expected benefit of avoiding treatment (31). Here, $P_{\mathrm{t}}$ was the threshold probability of LN metastasis.

The maximum Youden index was selected as the optimal cutoff value in the training cohort, and then, all 342 patients were divided into high-risk and low-risk subgroups. The sensitivity, specificity, accuracy, negative predictive value (NPV), and positive predictive value (PPV) of the nomogram were calculated in the training, validation, and total cohorts, respectively.

\section{Incremental value of the collagen signature to clinicopathological risk factors}

To evaluate the incremental value of the collagen signature to the clinicopathological risk factors, a clinicopathological model that included only the preoperative clinicopathological risk factors was constructed for comparison. The incremental value of the collagen signature in the clinicopathological model was assessed with respect to the AUROC and net benefits. 


\section{Association of the collagen signature-based nomogram with prognosis}

To determine the prognostic value of the collagen signaturebased nomogram, the associations between the nomogrampredicted LN metastasis and disease-free survival (DFS), recurrence-free survival (RFS) and overall survival (OS) were investigated for all patients. DFS was defined as the interval from surgery to first recurrence at any site or allcause death, whichever came first. RFS was defined as the interval from surgery to first recurrence. OS was defined as the interval between surgery and death or the last date of follow-up. The follow-up was censored on December 31, 2018.

\section{Statistical analysis}

Categorical variables were compared by using the chisquare test or Fisher's exact test. Continuous variables, where appropriate, were compared by using Student's $t$ test or the Mann-Whitney U test. Binary logistic regression analysis was used to calculate the odds ratio (OR) and the corresponding 95\% confidence interval (CI). Survival curves were generated by using the Kaplan-Meier method and compared by the log-rank test. Univariate and multivariate analyses with Cox proportional hazards regression determined the hazard ratio (HR) of preoperative predictors for DFS, RFS and OS. All statistical analyses were performed using R software (version 3.6.2) and SPSS (version 19.0), and a two-sided $\mathrm{P}<0.05$ was considered statistically significant. The LASSO regression analysis was performed using the "glmnet" package. The ROC curves were plotted, and the AUROC was calculated using the " $p R O C$ " package. The nomogram and calibration curve were generated using the "rms" package. The HosmerLemeshow test was conducted using the "generalhoslem" package. The decision curve analysis was performed using the function of " $d c a . R$ ", and the survival analysis was conducted using the "survminer" package.

\section{Results}

\section{Clinicopatbological characteristics}

The clinicopathological characteristics of the participants in the training $(\mathrm{N}=238)$ and validation $(\mathrm{N}=104)$ cohorts are summarized in Table 1. Of the 342 enrolled patients, $58.8 \%$ (201/342) were male, and the median (interquartile range,
IQR) age was 58 (48-65) years. The overall incidence of LN metastasis was $47.66 \%$ (163/342), with $47.48 \%$ $(113 / 238)$ in the training cohort and $48.07 \%(50 / 104)$ in the validation cohort $(\mathrm{P}=0.919)$. The diagnostic accuracy of the subjective CT-reported LN status was 59.06\% (202/342), with a sensitivity of $70.55 \%(115 / 163)$, a specificity of $48.60 \%$ (87/179), an NPV of 64.44\% (87/135), and a PPV of $55.56 \%(115 / 207)$.

\section{Collagen signature construction and its association with LN metastasis}

The framework for the construction of the collagen signature is presented in Figure 1. A seven-feature-based collagen signature was constructed from the 148 features using the LASSO regression method (Figure S2). The collagen signature was calculated using the following formula: collagen signature $=-1.70+0.25 \times$ mean of collagen number $+0.08 \times$ mean of cross-link density $+1.34 \times$ mean of collagen orientation $-0.30 \times$ variation of collagen orientation $0.005 \times$ kurtosis $+2.72 \times$ GLCM_constrast_O $0^{\circ} \_4$ pixel $+1.21 \times$ Gabor_mean_orientiation 1_scale 1 .

A significant difference in the collagen signature [median (IQR)] was found between patients with $\mathrm{LN}$ metastasis $[0.144(-0.208$ to 0.503$)]$ and patients without LN metastasis [-0.417 (-0.781 to -0.003$)]$ in the training cohort (median difference: 0.570 ; 95\% CI: $0.417-0.737$; $\mathrm{P}<0.001$ ) (Figure $2 A, B)$. This finding was confirmed in patients with LN metastasis $[0.273(-0.064$ to 0.994$)]$ and patients without LN metastasis [-0.415 (-0.761 to -0.019$)]$ in the validation cohort (median difference: $0.766 ; 95 \%$ CI, 0.548-1.041; $\mathrm{P}<0.001$ ) (Figure 2C,D). The collagen signature yielded an AUROC of 0.759 (95\% CI: 0.699 0.819 ) in the training cohort and 0.824 (95\% CI: $0.743-$ $0.904)$ in the validation cohort for $\mathrm{LN}$ metastasis, which indicated favorable predictive efficacy (Figure S3). Stratified analyses indicated that the collagen signature in patients with LN metastasis was also significantly higher than that in patients without LN metastasis under different preoperative variables in the training cohort, accompanied by satisfactory discrimination, which was also validated in the validation cohort (Table S2). In particular, in patients with negative CT-reported LN status (cN0), the collagen signature still showed a robust ability to discriminate the patients who were more likely to suffer from LN metastasis [AUROC: 0.788 (95\% CI: $0.692-0.885$ ) in the training cohort; 0.789 (95\% CI: 0.639-0.939) in the validation cohort]. 
Table 1 Characteristics of the participants in the training and validation cohorts

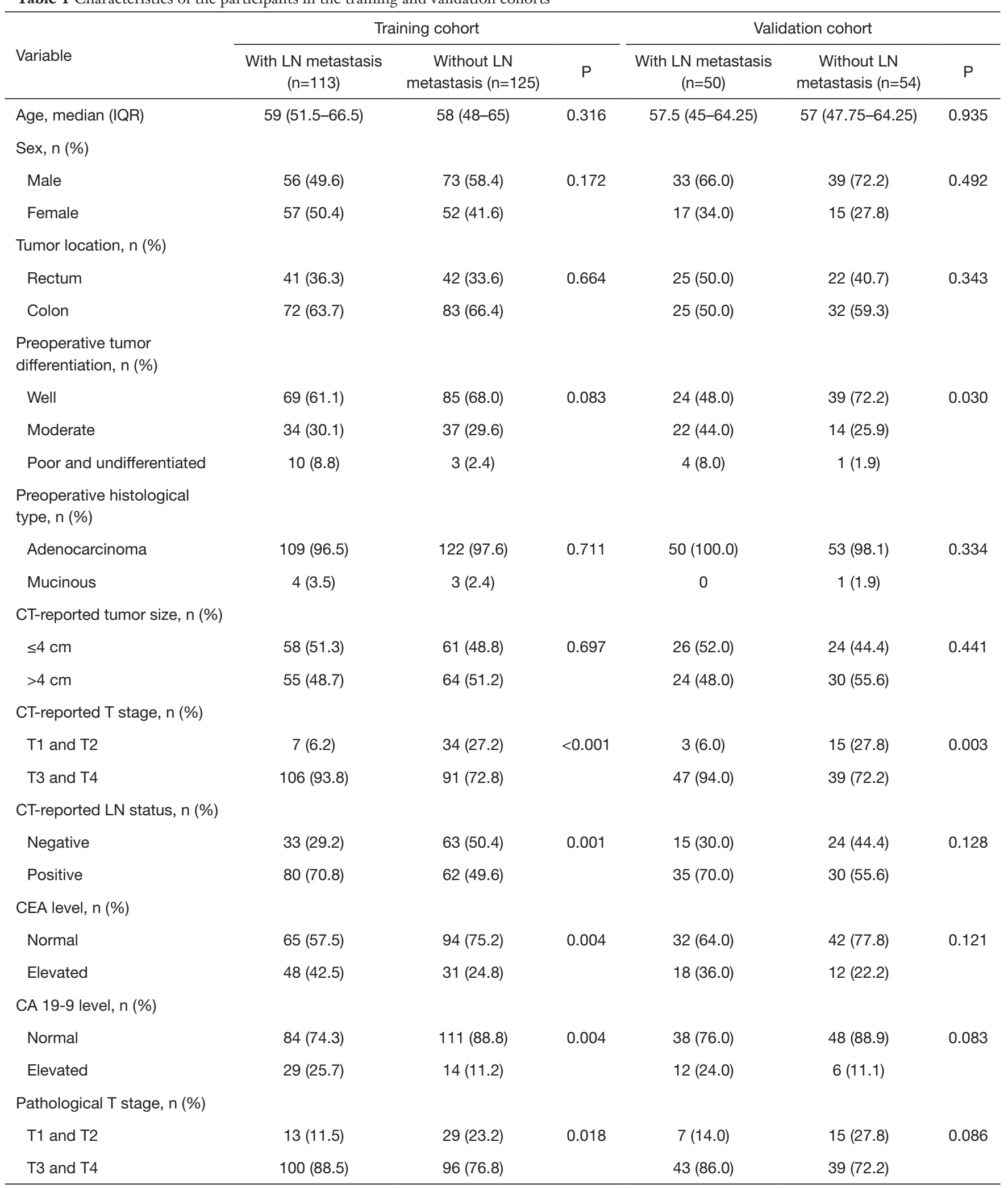

Table 1 (continued) 
Table 1 (continued)

\begin{tabular}{|c|c|c|c|c|c|c|}
\hline Variable & \multicolumn{3}{|c|}{ Training cohort } & \multicolumn{3}{|c|}{ Validation cohort } \\
\hline \multicolumn{7}{|l|}{$\begin{array}{l}\text { Postoperative tumor } \\
\text { differentiation, } \mathrm{n}(\%)\end{array}$} \\
\hline Well & $11(9.7)$ & $16(12.8)$ & 0.204 & $1(2.0)$ & $8(14.8)$ & 0.033 \\
\hline Poor and undifferentiated & $25(22.2)$ & $17(13.6)$ & & $11(22.0)$ & $6(11.1)$ & \\
\hline \multicolumn{7}{|l|}{$\begin{array}{l}\text { Postoperative histological } \\
\text { type, n (\%) }\end{array}$} \\
\hline Adenocarcinoma & $104(92.0)$ & $115(92.0)$ & 0.992 & $47(94.0)$ & $49(90.7)$ & 0.717 \\
\hline
\end{tabular}

IQR, interquartile range; CT, computed tomography; CEA, carcinoembryonic antigen; CA, carbohydrate antigen; LN, lymph node.

\section{Development, performance evaluation, and validation of the nomogram}

Univariate analyses were performed for each preoperative variable in the training cohort, among which the preoperative tumor differentiation ( $\mathrm{P}=0.046)$, CT-reported T stage $(\mathrm{P}<0.001)$, CT-reported $\mathrm{LN}$ status $(\mathrm{P}=0.001)$, CEA level $(\mathrm{P}=0.004)$, $\mathrm{CA}$ 19-9 level $(\mathrm{P}=0.005)$ and collagen signature $(\mathrm{P}<0.001)$ had significant associations with LN metastasis (Table 2). Compared with the other five predictors, the collagen signature showed the most powerful ability to predict LN status preoperatively (Figure S3). A prediction model was subsequently constructed based on the multivariate analysis of these preoperative predictors. The variance inflation factor of each predictor was less than 10 , with the corresponding tolerance exceeding 0.1 , indicating no multicollinearity among all predictors (Table S3) (26). A nomogram was developed on the basis of these six predictors (Figure $3 A$ ).

The nomogram demonstrated good accuracy in estimating the risk of LN metastasis (AUROC: 0.826, 95\% CI: 0.774-0.877) (Figure 3B). The calibration curve graphically indicated good agreement on the presence of LN metastasis between the risk estimation based on the nomogram and histopathologic findings on surgical specimens (Figure 3C). The Hosmer-Lemeshow test demonstrated a $\mathrm{P}$ value of 0.135 , indicating no departure from a good fit.
The abovementioned bootstrap method was used for internal validation, and the results remained largely unaltered between iterations, with a mean concordance index of 0.822. Good discrimination with an AUROC of 0.846 (95\% CI: $0.773-0.918$ ) was validated in the validation cohort, and a good calibration curve was also obtained for risk evaluation (Figure 3D,E). A Hosmer-Lemeshow test also yielded a nonsignificant $\mathrm{P}$ value of 0.766 .

\section{Clinical usefulness of the nomogram}

The decision curve analysis of the nomogram in the training and validation cohorts is depicted in Figure 4. The $x$ - and $y$-axes represent threshold probability and net benefit, respectively. The black and red lines represent the assumptions that no case and all cases had LN metastasis, respectively. The decision curve analysis demonstrated that using the collagen signature-based nomogram to detect the LN status could result in a higher net benefit than the treatall or treat-none scheme.

In addition, the maximum Youden index of 0.384 in the training cohort was selected as the optimal cutoff value, and then, all patients were divided into high-risk and low-risk subgroups. The sensitivity, specificity, accuracy, NPV and PPV of the nomogram in detecting the presence or absence of $\mathrm{LN}$ metastasis in the training cohort were $87.6 \%, 68.0 \%$, $77.3 \%, 86.2 \%$ and $71.4 \%$, respectively. In the validation 


\section{A Representative region of interest}

H\&E

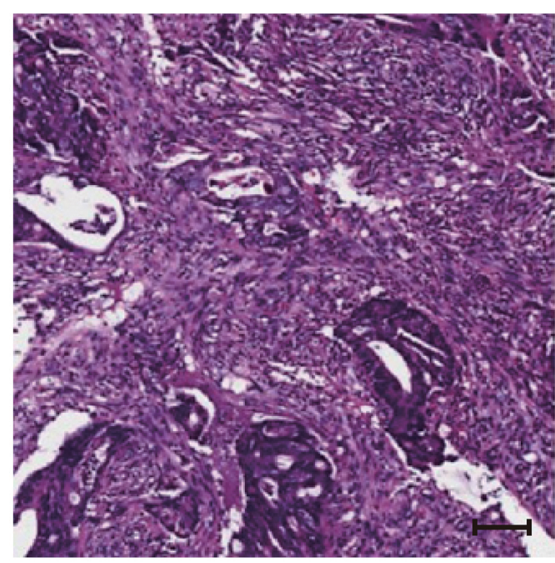

Multiphoton image

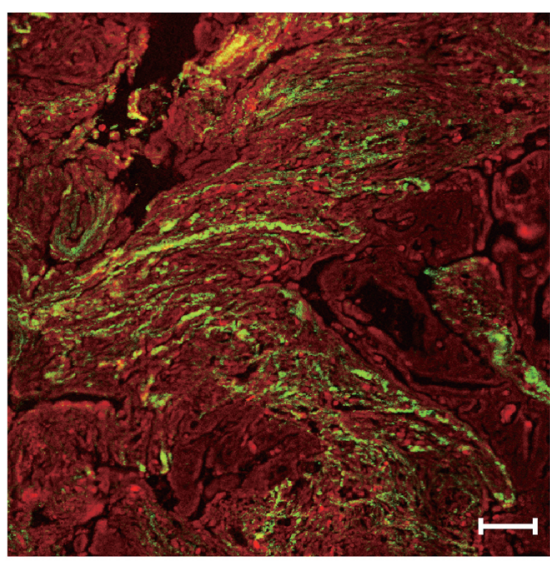

Binary mask image

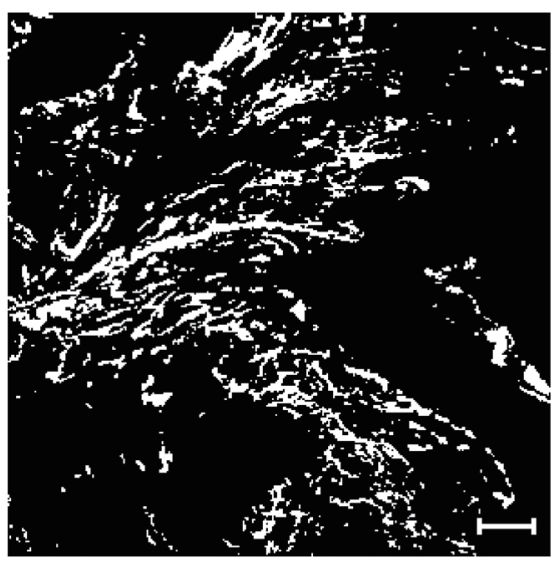

B

Framework of collagen signature construction

Region of interest

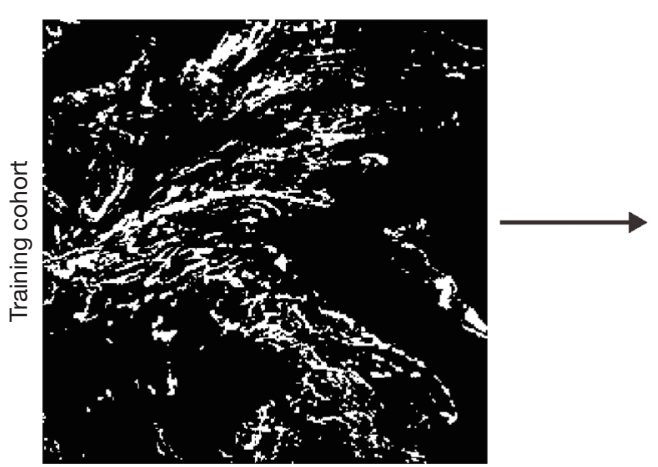

Features extraction

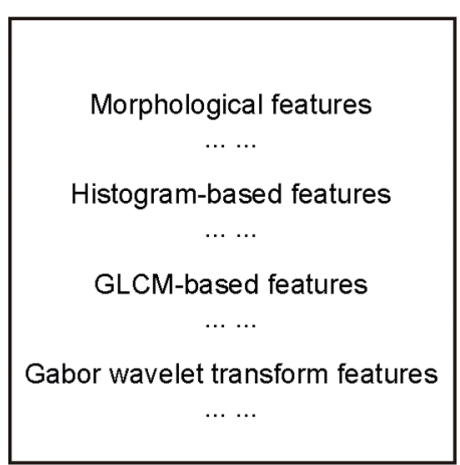

Features selection

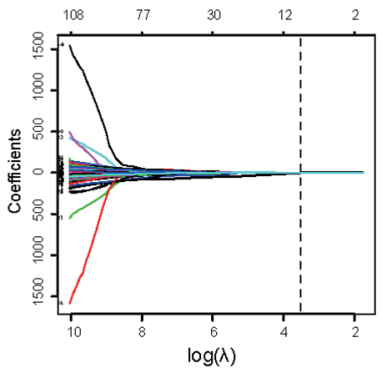

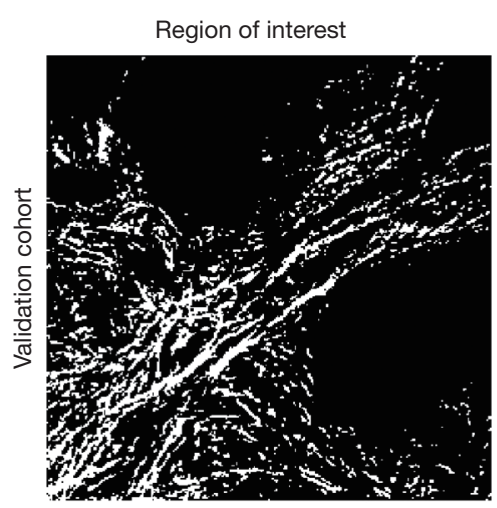

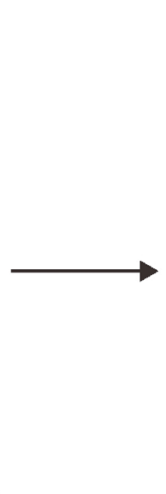

Features extraction

Morphological features
$\ldots \ldots$
Histogram-based features
$\ldots \ldots$
GLCM-based features
$\ldots \ldots$
Gabor wavelet transform features
$\ldots \ldots$

Collagen signature Collagen signature $=\Sigma \alpha_{i} \times E_{i}$

Figure 1 Schematic illustration of collagen signature construction. (A) A representative region of interest with an area of $500 \times 500 \mu \mathrm{m}$ was selected. The corresponding multiphoton image was obtained, and the SHG signal image was translated into a binary mask image for analysis. Scale bar: $50 \mu \mathrm{m}$. (B) A computational framework for collagen signature calculation. LASSO regression was used to select the predictive features in the training cohort, and then, a formula was built. The collagen signatures in both the training and validation cohorts were all calculated with the formula. H\&E, hematoxylin and eosin; SHG, second harmonic generation; LASSO, least absolute shrinkage and selection operator. 


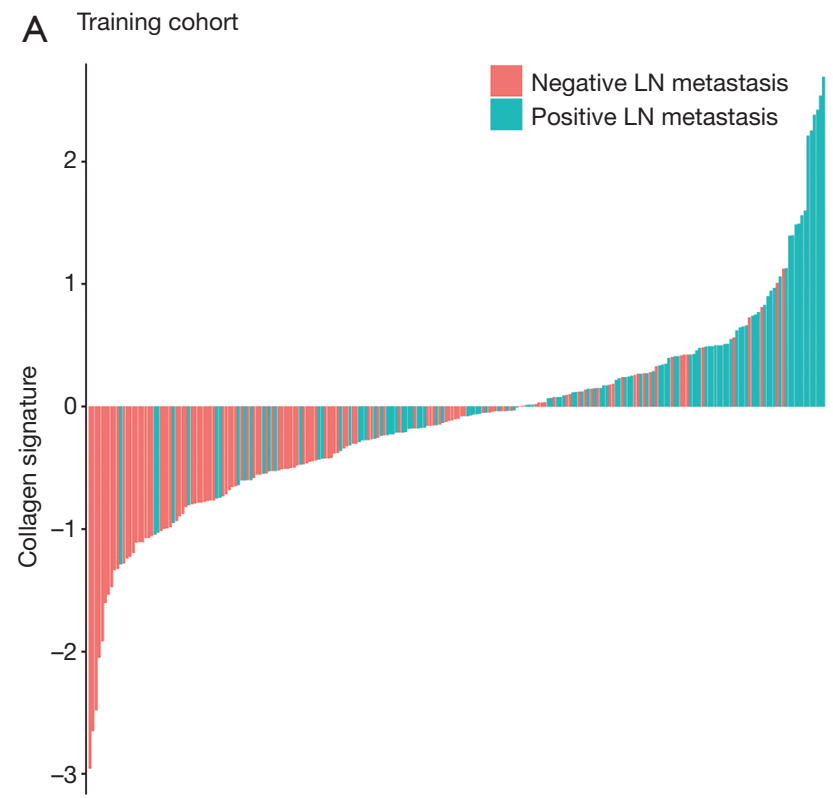

B Training cohort
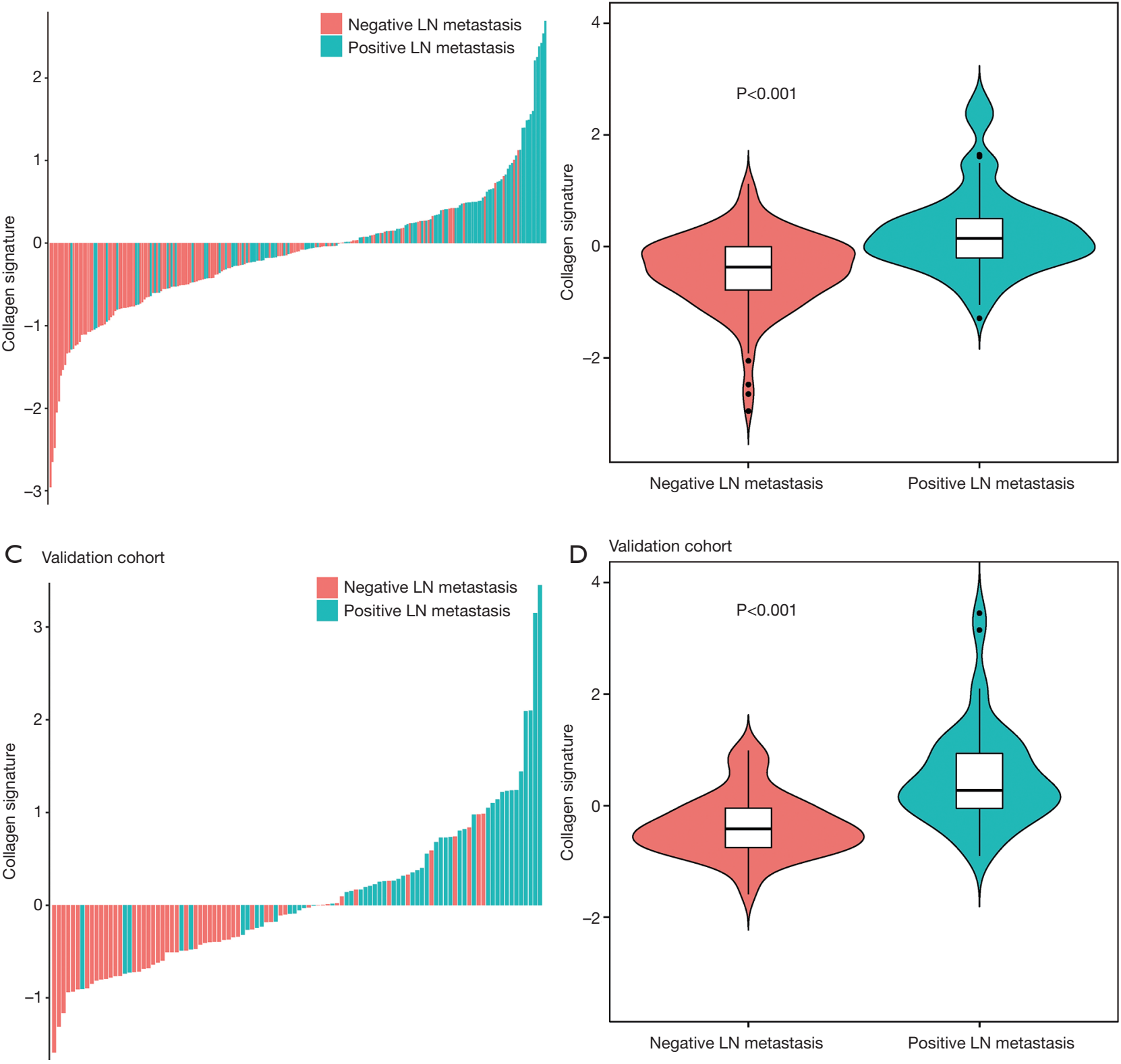

Figure 2 Distribution of the collagen signature and its relationship with LN metastasis. (A) Distribution of the collagen signature in the training cohort. (B) Comparison of the collagen signature between patients with and without LN metastasis in the training cohort. (C) Distribution of the collagen signature in the validation cohort. (D) Comparison of the collagen signature between patients with and without LN metastasis in the validation cohort. LN, lymph node.

cohort, the nomogram had a sensitivity of $84.0 \%$, a specificity of $70.4 \%$, an accuracy of $76.9 \%$, a NPV of $82.7 \%$ and a PPV of $72.7 \%$. Among all 342 patients, the sensitivity was $86.5 \%$, the specificity was $68.2 \%$, the accuracy was $76.9 \%$, the NPV was $84.9 \%$, and the PPV was $71.2 \%$ (Table 3).

\section{Incremental value of the collagen signature to clinicopathological risk factors}

To assess the incremental value of the collagen signature to clinicopathological risk factors, we excluded the collagen signature and constructed a clinicopathological model 
Table 2 Univariate and multivariate logistic analyses in the training cohort

\begin{tabular}{|c|c|c|c|c|}
\hline Variable & \multicolumn{2}{|c|}{ Univariate analysis } & \multicolumn{2}{|c|}{ Multivariate analysis } \\
\hline Age & $0.992(0.972-1.013)$ & 0.458 & - & - \\
\hline Sex (female vs. male) & $1.429(0.856-2.385)$ & 0.172 & - & - \\
\hline Location (colon vs. rectum) & $0.889(0.521-1.515)$ & 0.665 & - & - \\
\hline Preoperative tumor differentiation & & 0.046 & & 0.091 \\
\hline Well & Reference & $>0.99$ & Reference & $>0.99$ \\
\hline Moderate & $1.132(0.644-1.989)$ & 0.666 & $1.025(0.514-2.040)$ & 0.945 \\
\hline CT-reported LN status (positive vs. negative) & $2.463(1.441-4.210)$ & 0.001 & $2.392(1.233-4.641)$ & 0.010 \\
\hline CEA level (elevated vs. normal) & $2.239(1.290-3.886)$ & 0.004 & $1.698(0.838-3.439)$ & 0.141 \\
\hline CA 19-9 level (elevated vs. normal) & $2.737(1.362-5.501)$ & 0.005 & $2.123(0.873-5.160)$ & 0.097 \\
\hline Collagen signature & $5.426(3.107-9.476)$ & $<0.001$ & $5.950(3.175-11.152)$ & $<0.001$ \\
\hline
\end{tabular}

OR, odd ratio; $\mathrm{Cl}$, confidence interval; CT, computed tomography; CEA, carcinoembryonic antigen; CA, carbohydrate antigen; LN, lymph node.

based on preoperative tumor differentiation, CT-reported T stage, CT-reported LN status, CEA level and CA 19-9 level (Table S4). The clinicopathological model showed AUROCs of 0.726 (95\% CI: 0.662-0.798) and 0.727 (95\% CI: $0.623-0.824)$ in the training and validation cohorts, respectively (Figure S4).

Compared with the clinicopathological model, the collagen signature-based nomogram, which included the collagen signature in the clinicopathological model, exhibited a significant improvement in discriminative ability for LN metastasis (Table S5, Figure S5), with an increased AUROC of 0.832 (95\% CI: 0.789-0.874), which was over 0.726 (95\% CI: 0.671-0.778), for all 342 patients $(\mathrm{P}<0.001)$. The decision curve analysis also indicated that the net benefit would be higher with the collagen signature-based nomogram than with the clinicopathological model for estimating the risk of LN metastasis (Figure 4).

\section{Association between the collagen signature-based nomogram and prognosis}

The median follow-up time of all enrolled patients was 21
(IQR: 13.75-37) months, with an estimated 3-year DFS of $68.9 \%$ (95\% CI: $63.1-75.4 \%$ ), 3-year DFS of $70.6 \%(95 \%$ CI: $64.7-77.0 \%)$ and 3 -year OS of 79.3\% (95\% CI: 73.6$85.3 \%$ ) (Figure S6).

Among patients in the nomogram-predicted high-risk subgroup, DFS was significantly worse than that among patients in the nomogram-predicted low-risk subgroup [3-year DFS: high risk, 56.2\% (95\% CI: 47.6-66.2\%); low risk, 84.7\% (78.0-92.1\%); log-rank $\mathrm{P}<0.001]$ (Figure $5 A$ ). Additionally, a worse RFS was observed in patients with high-risk subgroup compared to patients with low-risk subgroup [3-year RFS: high risk, 57.9\% (95\% CI: 49.368.1\%); low risk: $86.1 \%$ (95\% CI: 79.4-93.3\%); log-rank $\mathrm{P}<0.001$ ] (Figure 5B). Similarly, the OS of patients in the high-risk subgroup was worse than that of the patients in the low-risk subgroup [3-year OS: high risk, 68.4\% (95\% CI: 59.6-78.5\%); low risk, 90.3\% (84.2-97.0\%); log-rank $\mathrm{P}<0.001$ ] (Figure 5C). Univariate Cox regression analysis revealed that the nomogram-predicted high-risk subgroup was significantly associated with an unfavorable DFS (HR: 3.345, 95\% CI: 1.978-5.656, P<0.001), RFS (HR: 3.567, 95\% CI: 2.050-6.207; P<0.001) and OS (HR: 3.763, 95\% 
A

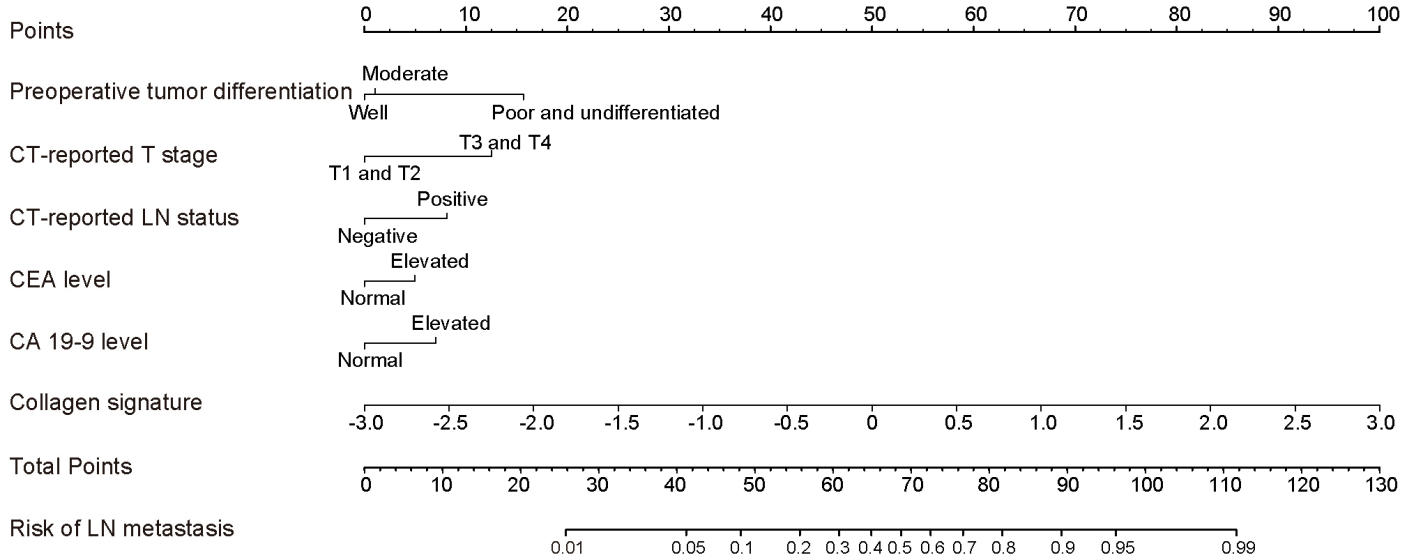

B

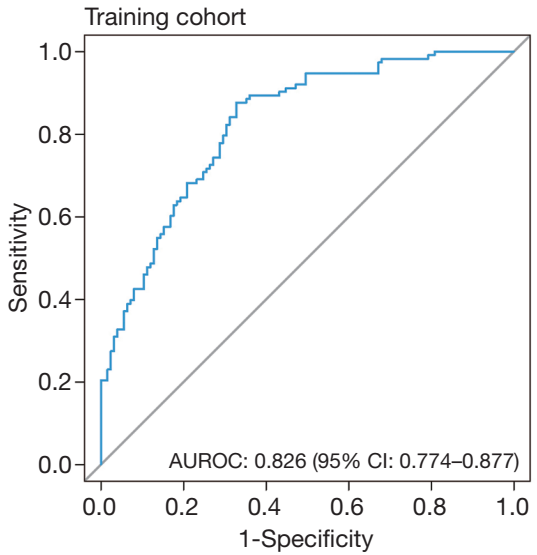

D

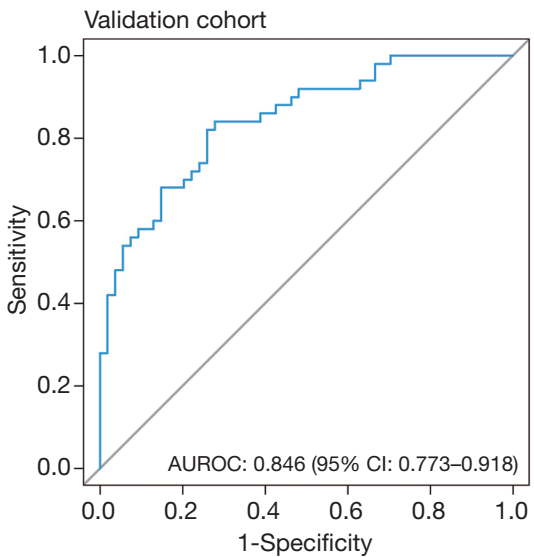

C

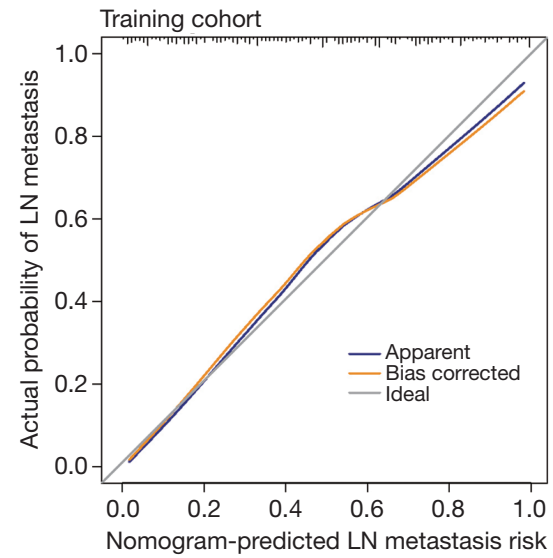

$E$

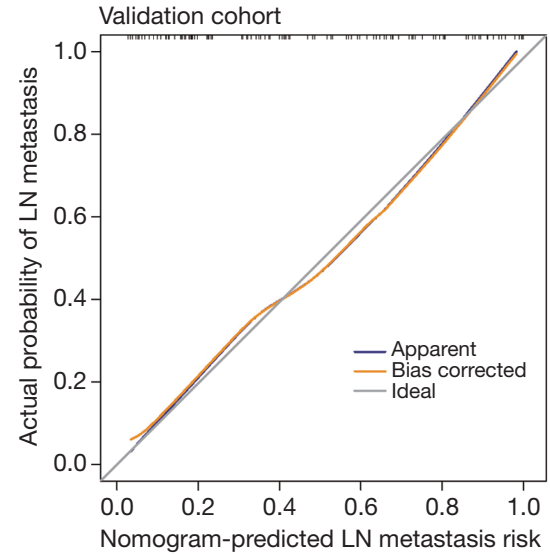

Figure 3 Nomogram and performance evaluation. (A) Newly developed collagen signature-based nomogram. (B) ROC curve of the nomogram in training cohort. (C) Calibration curve of the nomogram in the training cohort. (D) ROC curve of the nomogram in the validation cohort. (E) Calibration curve of the nomogram in the validation cohort. For clinical use, tumor differentiation is determined by drawing a line straight up to the point axis to establish the score associated with preoperative tumor differentiation. Then, this process is repeated for the other five covariates. The scores of each covariate are added, and the total score is located on the total score points axis. Finally, a line is drawn straight down to the risk of the LN metastasis axis to obtain the probability. In the calibration curve, the y-axis represents the actual LN metastasis rate, and the $\mathrm{x}$-axis represents the nomogram-predicted LN metastasis probability. The diagonal gray line represents a perfect prediction using an ideal model. The blue line represents the performance of the nomogram. The orange line represents the bias-corrected performance of the nomogram. CT, computed tomography; CEA, carcinoembryonic antigen; CA, carbohydrate antigen; LN, lymph node; ROC, receiver operator characteristic; AUROC, area under the receiver operator characteristic curve. 

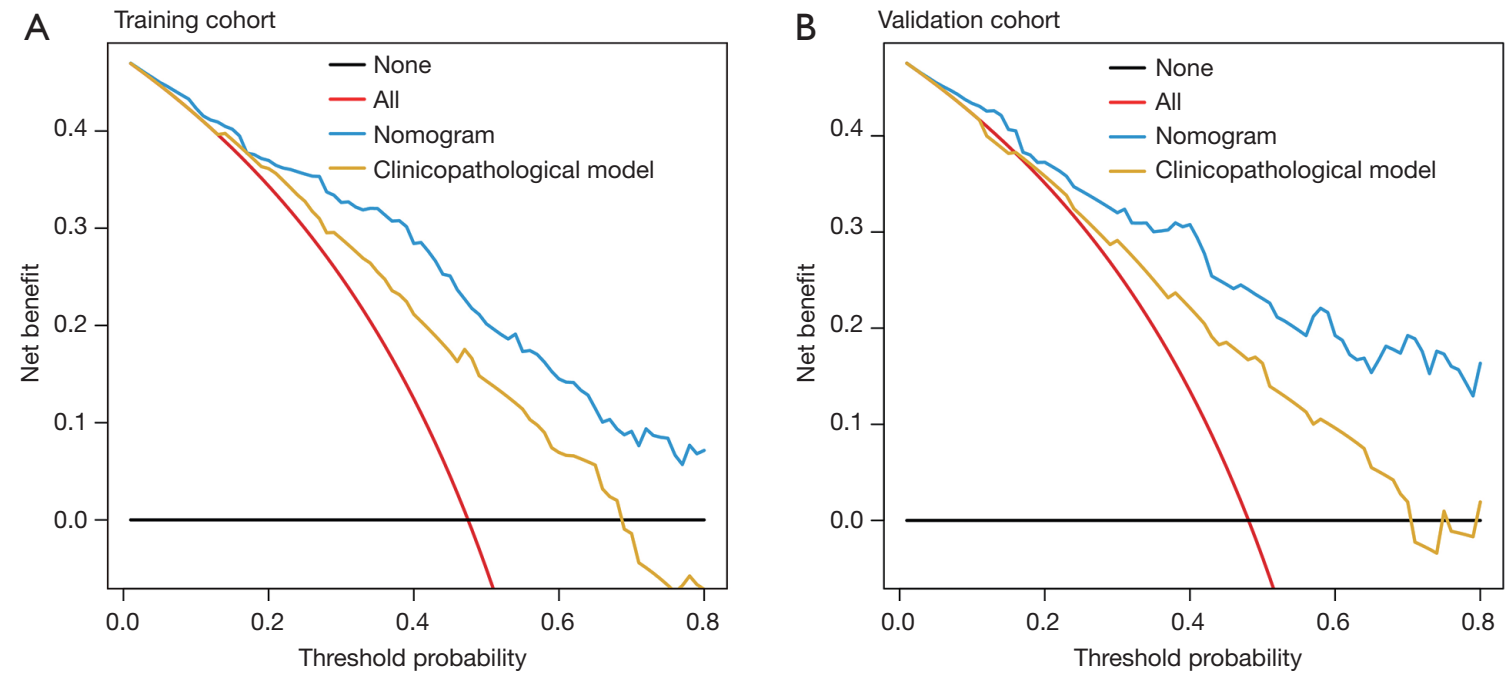

Figure 4 Decision curve analysis. (A) Decision curve analysis of the training cohort. (B) Decision curve analysis of the validation cohort. The red line and black line represent the assumption regarding all patients with LN metastasis and all patients without LN metastasis, respectively. The blue line represents the collagen signature-based nomogram, and the yellow line represents the clinicopathological model. LN, lymph node.

Table 3 Diagnostic performance of the nomogram in estimating the risk of $\mathrm{LN}$ metastasis

\begin{tabular}{|c|c|c|c|}
\hline Variable & \multicolumn{3}{|c|}{ Value $(95 \% \mathrm{Cl})$} \\
\hline Cutoff value & 0.384 & 0.384 & 0.384 \\
\hline Sensitivity, \% & $87.6(70.8-93.8)$ & $84.0(74.0-94.0)$ & $86.5(81.0-91.4)$ \\
\hline Specificity, \% & $68.0(58.4-82.4)$ & $70.4(57.4-81.5)$ & $68.2(61.5-74.9)$ \\
\hline Negative predictive value, $\%$ & $86.2(75.5-92.5)$ & 82.7 (73.2-92.3) & $84.9(79.5-89.9)$ \\
\hline Positive predictive value, $\%$ & $71.4(65.8-79.1)$ & 72.7 (64.2-81.5) & $71.2(66.7-75.9)$ \\
\hline
\end{tabular}

$\mathrm{Cl}$, confidence interval; LN, lymph node.

CI: 1.876-7.545; $\mathrm{P}<0.001)$. Nomogram-predicted LN metastasis remained an independent preoperative predictor of DFS and OS even upon adjustment for the preoperative clinicopathological risk factors (Table S6). In addition, in 77 patients who suffered from recurrence, 32 patients were diagnosed as local recurrence and 45 patients were diagnosed as distant metastasis, respectively. Among the 32 patients with local recurrence, the percentage of low- and high-risk subgroup were $18.8 \%$ (6/32) and $81.2 \%(26 / 32)$, respectively. In the 45 patients with distant metastasis, $22.2 \%(10 / 45)$ patients were low-risk subgroup, and $77.8 \%$ (35/45) patients were high-risk subgroup (Figure S7).
No significant difference was found between pattern of recurrence and nomogram-predicted subgroup $(\mathrm{P}=0.711)$.

\section{Discussion}

Accurate estimation of the risk of LN metastasis in CRC before surgery is vital for decision-making and prognostic predictions. In this study, we built a multi-featurebased collagen signature from preoperative biopsies, and this signature showed a significant association with LN metastasis. Additionally, we developed and validated a collagen signature-based nomogram that showed a robust 

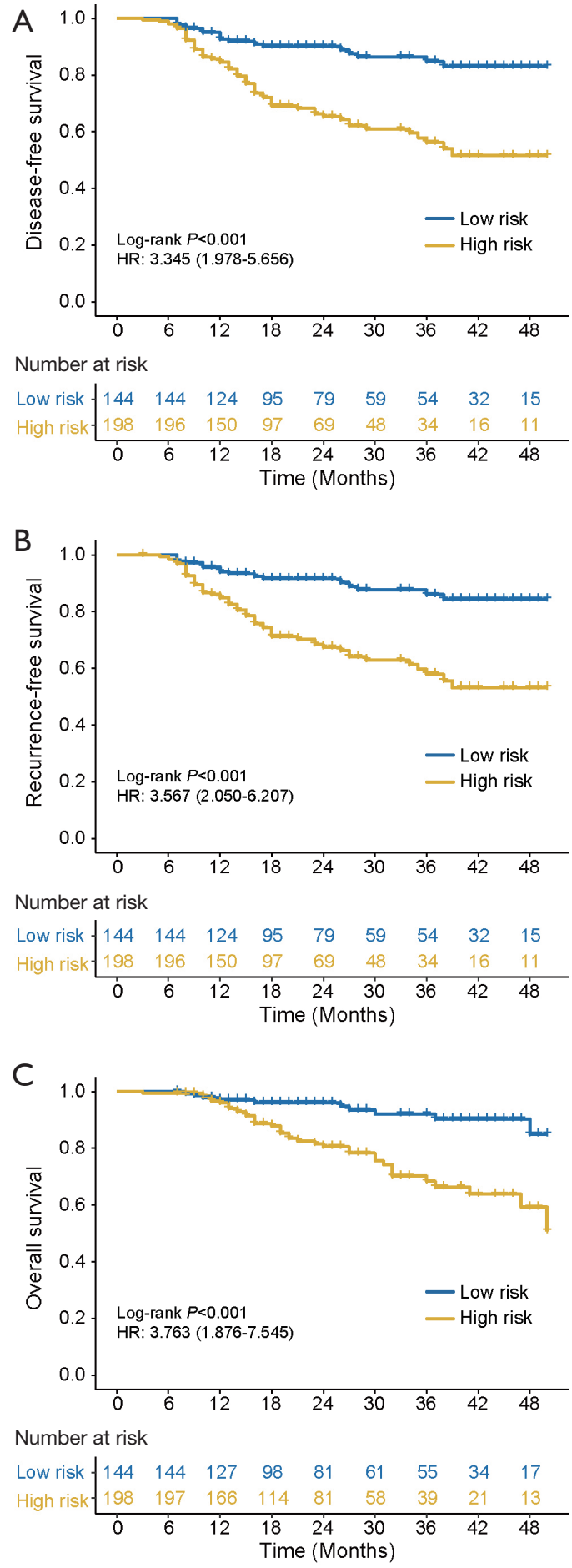

Figure 5 Kaplan-Meier analysis of disease-free survival, recurrencefree survival and overall survival according to the nomogram-predicted subgroups of all patients. (A) Disease-free survival of all patients in the high- and low-risk subgroups. (B) Recurrence-free survival of all patients in the high- and low-risk subgroups. (C) Overall survival of all patients in the high- and low-risk subgroups. ability to predict LN status. Furthermore, our decision curve analysis revealed that the net benefits would be higher with the nomogram than with the treat-all or treat-none scheme. Compared with that of the clinicopathological model, the predictive performance of the collagen signature-based nomogram was improved. In addition, the nomogram prediction of $\mathrm{LN}$ metastasis was relevant to patient prognosis and was an independent preoperative prognostic predictor for DFS and OS.

Collagen in the tumor microenvironment is considered a promising biomarker with great potential for clinical translation in the era of personalized medicine $(7,32)$. However, despite some advances in the application of collagen in the clinic, most studies have been based on qualitative or semiquantitative results $(9,33,34)$. To our knowledge, this is the first analysis of the predictive biomarker for $\mathrm{LN}$ metastasis based on the fully quantitative collagen signature obtained from the multiphoton images of preoperative biopsies in patients with CRC.

There were two main factors that determined the construction of the collagen signature. The first was an appropriate imaging method for specific visualization of collagen. Herein, multiphoton imaging was used because of its underlying physical origin $(11,13)$. The second was a fully quantitative approach to comprehensively evaluate collagen alterations in the tumor microenvironment. For this purpose, we have established a framework for the quantification of collagen $(8,14,15)$. Thus, it is feasible to construct the collagen signature of biopsies in CRC.

Currently, even with the combination of CT with other imaging examinations, the overall accuracy of CT for the preoperative evaluation of LN metastasis is not more than $60 \%$ (4). In our study, the diagnostic accuracy of CT for LN status was only $59.06 \%$, with a sensitivity of $70.55 \%$ and a specificity of $48.60 \%$. The unfavorable performance for nodal staging constantly resulted in underor overtreatment. According to the collagen signaturebased nomogram, the overall accuracy for nodal staging was increased to $76.9 \%$, with improved sensitivity and specificity values of $86.5 \%$ and $68.2 \%$, respectively, which would favor clinical decision-making regarding the treatment of CRC.

Compared to patients with a nomogram-predicted low risk of $\mathrm{LN}$ metastasis, those with a high risk of LN metastasis had greatly decreased predicted DFS and OS rates, even upon receiving curative-intent surgery. The high-risk patients had a 3.345 -fold increased risk of a poor DFS and a 3.763-fold increased risk of a poor OS compared 
to low-risk patients. Multivariable Cox regression analyses also indicated that the nomogram-predicted LN status was an independent prognostic predictor of survival. In these high-risk cases, neoadjuvant chemotherapy might be considered for the potential improvement of survival (35).

To improve the predictive performance of $\mathrm{LN}$ metastasis in CRC, researchers have discovered several biomarkers. $\mathrm{Qu}$ et al. (36) constructed a miRNA panel based on four differentially expressed miRNAs, namely, miR-122-5p, miR-146b-5p, miR-186-5p and miR-193a-5p, in serum samples of CRC patients and found that the miRNA panel could increase the LN prediction capability compared with CT. Furthermore, a nomogram encompassing the miRNA panel and CT-reported LN status was developed, and this nomogram performed well in predicting the LN status. Huang et al. (37) presented a radiomics nomogram that incorporated the radiomics signature and other preoperative risk factors involving the CT-reported $\mathrm{LN}$ status and CEA, with a desirable AUROC of 0.736 . Wei et al. (38) established a gene-related nomogram, which included 59 hub genes, for the prediction of LN metastasis in CRC and demonstrated its good diagnostic value. Although these biomarkers have not yet been translated to clinical application, we still envision that various biomarkers from radiomics, histology, serology and genomics can be considered together to improve the accuracy of estimating the risk of LN metastasis in CRC preoperatively in the future.

Multiphoton imaging is a useful approach for visualizing the tissue structure and cell morphology of samples based on their intrinsic signals without the need for additional fluorescent dyes $(11,39)$. Additionally, multiphoton imaging provides important information on stromal collagen when its features are robustly measured and quantified (40). Because of the comparable results of multiphoton imaging to those of $\mathrm{H} \& \mathrm{E}$ staining, it is possible for investigators to define the regions of interest using only multiphoton imaging, after training (39). Considering the negligible effect of tissue fixation and paraffin embedding, additional removal of the paraffin in biopsies is not needed, as it would not affect multiphoton imaging (41). Multiphoton imaging of each biopsy takes approximately ten minutes, and the collagen signature can be calculated from the formula; therefore, this approach does not significantly increase the work burden imposed on clinicians.

Nomograms are widely used as predictive or prognostic tools in oncology. They have the ability to generate an individual probability of a clinical event by integrating diverse predictive or prognostic variables, thereby meeting the need for biologically and clinically integrated models and getting us closer to our goal of precision medicine (42). In this study, the following six predictors always contributed to the estimation of LN metastasis: preoperative tumor differentiation, CT-reported T stage, CT-reported LN status, CEA level and CA 19-9 level. These data were obtained from routine examinations in the clinic, and the collagen signature could be acquired after multiphoton imaging, which made calculating the individual risk of LN metastasis convenient.

There are three aspects where the nomogram might change the approach to therapy of CRC. First, the feasibility and potential survival benefits from neoadjuvant chemotherapy for locally advanced colon cancer have been proved, thus, patients with nomogram-predicted high risk of LN metastasis might also be candidates for neoadjuvant chemotherapy $(43,44)$. Second, for rectal cancer, patients with nomogram-predicted high risk of LN metastasis would be suggested to receive preoperative chemoradiotherapy. Third, for submucosally invasive colorectal cancer (T1), additional surgical resection might be required for patients with nomogram-predicate high risk of LN metastasis after endoscopic resection.

Despite the satisfactory ability of the collagen signaturebased nomogram to predict LN status, some limitations in our study should not be ignored. First, due to the retrospective nature of this study, potential selection bias was inevitable. Thus, a prospective CRC cohort is needed to verify the performance of the nomogram. Second, all enrolled participants came from a single institution. Hence, cohorts from other medical centers, especially from those in Western countries, are needed to further validate these findings.

In conclusion, this study revealed that the fully quantitative collagen signature in the tumor microenvironment of preoperative biopsies is an independent predictor for $\mathrm{LN}$ metastasis in CRC. Additionally, the collagen signaturebased nomogram we developed and validated is helpful for individual estimations of the risk of LN metastasis among patients with CRC. Moreover, the nomogram is useful for prognostic predictions before surgery, which might facilitate decision-making and improve survival among CRC patients.

\section{Acknowledgments}

Funding: This study was supported by grants from the 
National Natural Science Foundation of China (Grant No. 81773117, 81771881, and 81772964), the Special Scientific Research Fund of Public Welfare Profession of National Health and Family Planning Commission (Grant No. 201502026), the Guangdong Gastrointestinal Disease Research Center (Grant No. 2017B020209003), the Guangdong Provincial Key Laboratory of Precision Medicine for Gastrointestinal Cancer (Grant No. 2020B121201004), the Natural Science Foundation of Guangdong Province (Grant No. 2020A1515010035), the Clinical Research Project of Nanfang Hospital (Grant No. 2018CR034, 2020CR001 and 2020CR011), and the China Postdoctoral Science Foundation (Grant No. 2020M682789).

\section{Footnote}

Reporting Checklist: The authors have completed the TRIPOD reporting checklist. Available at http://dx.doi. org/10.21037/atm-20-7565

Data Sharing Statement: Available at http://dx.doi. org/10.21037/atm-20-7565

Conflicts of Interest: All authors have completed the ICMJE uniform disclosure form (available at http://dx.doi. org/10.21037/atm-20-7565). The authors have no conflicts of interest to declare.

Ethical Statement: The authors are accountable for all aspects of the work in ensuring that questions related to the accuracy or integrity of any part of the work are appropriately investigated and resolved. This retrospective study was approved by the institutional review board of Nanfang Hospital, Southern Medical University, which waived the requirement to obtain written informed consent and was carried out according to the Declaration of Helsinki (as revised in 2013) for biomedical research involving human subjects.

Open Access Statement: This is an Open Access article distributed in accordance with the Creative Commons Attribution-NonCommercial-NoDerivs 4.0 International License (CC BY-NC-ND 4.0), which permits the noncommercial replication and distribution of the article with the strict proviso that no changes or edits are made and the original work is properly cited (including links to both the formal publication through the relevant DOI and the license). See: https://creativecommons.org/licenses/by-nc-nd/4.0/.

\section{References}

1. Bray F, Ferlay J, Soerjomataram I, et al. Global cancer statistics 2018: GLOBOCAN estimates of incidence and mortality worldwide for 36 cancers in 185 countries. CA Cancer J Clin 2018;68:394-424.

2. Hashiguchi Y, Muro K, Saito Y, et al. Japanese Society for Cancer of the Colon and Rectum (JSCCR) guidelines 2019 for the treatment of colorectal cancer. Int J Clin Oncol 2020;25:1-42.

3. Glasgow SC, Bleier JIS, Burgart LJ, et al. Meta-analysis of histopathological features of primary colorectal cancers that predict lymph node metastases. J Gastrointest Surg 2012;16:1019-28.

4. Brouwer NP, Stijns R, Lemmens V, et al. Clinical lymph node staging in colorectal cancer; a flip of the coin? Eur J Surg Oncol 2018;44:1241-6.

5. Theocharis AD, Skandalis SS, Gialeli C, et al. Extracellular matrix structure. Adv Drug Deliv Rev 2016;97:4-27.

6. Yamauchi M, Barker TH, Gibbons DL, et al. The fibrotic tumor stroma. J Clin Invest 2018;128:16-25.

7. $\mathrm{Xu} \mathrm{S}, \mathrm{Xu} \mathrm{H}$, Wang $\mathrm{W}$, et al. The role of collagen in cancer: from bench to bedside. J Transl Med 2019;17:309.

8. Chen D, Chen G, Jiang W, et al. Association of the collagen signature in the tumor microenvironment with lymph node metastasis in early gastric cancer. Jama Surg 2019;154:e185249.

9. Toss MS, Miligy IM, Gorringe KL, et al. Geometric characteristics of collagen have independent prognostic significance in breast ductal carcinoma in situ: an image analysis study. Mod Pathol 2019;32:1473-85.

10. Saito T, Uzawa K, Terajima M, et al. Aberrant collagen cross-linking in human oral squamous cell carcinoma. J Dent Res 2019;98:517-25.

11. Zipfel WR, Williams RM, Christie R, et al. Live tissue intrinsic emission microscopy using multiphoton-excited native fluorescence and second harmonic generation. Proc Natl Acad Sci U S A 2003;100:7075-80.

12. Chen X, Nadiarynkh O, Plotnikov S, et al. Second harmonic generation microscopy for quantitative analysis of collagen fibrillar structure. Nat Protoc 2012;7:654-69.

13. Campagnola P. Second harmonic generation imaging microscopy: applications to diseases diagnostics. Anal Chem 2011;83:3224-31. 


\section{Page 16 of 17}

14. Xu S, Wang Y, Tai D, et al. qFibrosis: a fully-quantitative innovative method incorporating histological features to facilitate accurate fibrosis scoring in animal model and chronic hepatitis B patients. J Hepatol 2014;61:260-9.

15. Xu S, Kang CH, Gou X, et al. Quantification of liver fibrosis via second harmonic imaging of the Glisson's capsule from liver surface. J Biophotonics 2016;9:351-63.

16. Zhang JX, Song W, Chen ZH, et al. Prognostic and predictive value of a microRNA signature in stage II colon cancer: a microRNA expression analysis. Lancet Oncol 2013;14:1295-306.

17. Jiang Y, Zhang Q, Hu Y, et al. ImmunoScore signature: a prognostic and predictive tool in gastric cancer. Ann Surg 2018;267:504-13.

18. Tibshirani R. Regression shrinkage and selection via the lasso: a retrospective. J R Stat Soc B 2011;73:273-82.

19. Ranstam J, Cook JA. LASSO regression. Br J Surg 2018;105:1348.

20. Zhuo S, Chen J, Luo T, et al. Multimode nonlinear optical imaging of the dermis in ex vivo human skin based on the combination of multichannel mode and Lambda mode. Opt Express 2006;14:7810-20.

21. Dempster A, Laird N, Rubin D. Maximum likelihood from incomplete data via the EM algorithm. J. Royal Statistical Society, Series B 1977;39:1-38.

22. Stein AM, Vader DA, Jawerth LM, et al. An algorithm for extracting the network geometry of three-dimensional collagen gels. J Microsc 2008;232:463-75.

23. Frisch KE, Duenwald-Kuehl SE, Kobayashi H, et al. Quantification of collagen organization using fractal dimensions and Fourier transforms. Acta Histochem 2012;114:140-4.

24. Haralick RM, Shanmugam K, Dinstein I. Textural features for image classification. IEEE Trans Syst Man Cybern 1973;3:610-21.

25. Daugman GJ. Complete discrete 2-D Gabor transforms by neural networks for image analysis and compression. IEEE Trans Acoust 1988;36:1169-79.

26. Dormann CF, Elith J, Bacher S, et al. Collinearity: a review of methods to deal with it and a simulation study evaluating their performance. Ecography 2013;36:27-46.

27. Collins GS, Reitsma JB, Altman DG, et al. Transparent Reporting of a multivariable prediction model for Individual Prognosis or Diagnosis (TRIPOD). Ann Intern Med 2015;162:735-6.

28. Kramer AA, Zimmerman JE. Assessing the calibration of mortality benchmarks in critical care: The HosmerLemeshow test revisited. Crit Care Med 2007;35:2052-6.
Fu et al. Collagen signature predicts LN status of CRC preoperatively

29. Iasonos A, Schrag D, Raj GV, et al. How to build and interpret a nomogram for cancer prognosis. J Clin Oncol 2008;26:1364-70.

30. Vickers AJ, Elkin EB. Decision curve analysis: a novel method for evaluating prediction models. Med Decis Making 2006;26:565-74.

31. Kerr KF, Brown MD, Zhu K, et al. Assessing the clinical impact of risk prediction models with decision curves: guidance for correct interpretation and appropriate use. J Clin Oncol 2016;34:2534-40.

32. Martins Cavaco AC, Damaso S, Casimiro S, et al. Collagen biology making inroads into prognosis and treatment of cancer progression and metastasis. Cancer Metastasis Rev 2020;39:603-23.

33. Ma HP, Chang HL, Bamodu OA, et al. Collagen 1A1 (COL1A1) is a reliable biomarker and putative therapeutic target for hepatocellular carcinogenesis and metastasis. Cancers (Basel) 2019;11:786.

34. Conklin MW, Eickhoff JC, Riching KM, et al. Aligned collagen is a prognostic signature for survival in human breast carcinoma. Am J Pathol 2011;178:1221-32.

35. Jin M, Frankel WL. Lymph node metastasis in colorectal cancer. Surg Oncol Clin N Am 2018;27:401-12.

36. Qu A, Yang Y, Zhang X, et al. Development of a preoperative prediction nomogram for lymph node metastasis in colorectal cancer based on a novel serum miRNA signature and CT scans. Ebiomedicine 2018;37:125-33.

37. Huang YQ, Liang CH, He L, et al. Development and validation of a radiomics nomogram for preoperative prediction of lymph node metastasis in colorectal cancer. J Clin Oncol 2016;34:2157-64.

38. Wei S, Zang J, Jia Y, et al. A gene-related nomogram for preoperative prediction of lymph node metastasis in colorectal cancer. J Invest Surg 2020;33:715-22.

39. Yan J, Zheng X, Liu Z, et al. Multiphoton imaging provides a superior optical biopsy to that of confocal laser endomicroscopy imaging for colorectal lesions. Endoscopy 2019;51:174-8.

40. Westreich J, Khorasani M, Jones B, et al. Novel methodology to image stromal tissue and assess its morphological features with polarized light: towards a tumour microenvironment prognostic signature. Biomed Opt Express 2019;10:3963-73.

41. Kakkad SM, Solaiyappan M, Argani P, et al. Collagen I fiber density increases in lymph node positive breast cancers: pilot study. J Biomed Opt 2012;17:116017.

42. Balachandran VP, Gonen M, Smith JJ, et al. Nomograms 
in oncology: more than meets the eye. Lancet Oncol 2015;16:e173-80.

43. Cheong CK, Nistala $\mathrm{K}, \mathrm{Ng} \mathrm{CH}$, et al. Neoadjuvant therapy in locally advanced colon cancer: a meta-analysis and systematic review. J Gastrointest Oncol 2020;11:847-57.

Cite this article as: Fu M, Chen D, Luo F, Wang G, Xu S, Wang Y, Sun C, Xu X, Li A, Zhuo S, Liu S, Yan J. Development and validation of a collagen signature-based nomogram for preoperatively predicting lymph node metastasis and prognosis in colorectal cancer. Ann Transl Med 2021;9(8):651. doi: 10.21037/atm-20-7565
44. Foxtrot Collaborative Group. Feasibility of preoperative chemotherapy for locally advanced, operable colon cancer: the pilot phase of a randomised controlled trial. Lancet Oncol 2012;13:1152-60. 


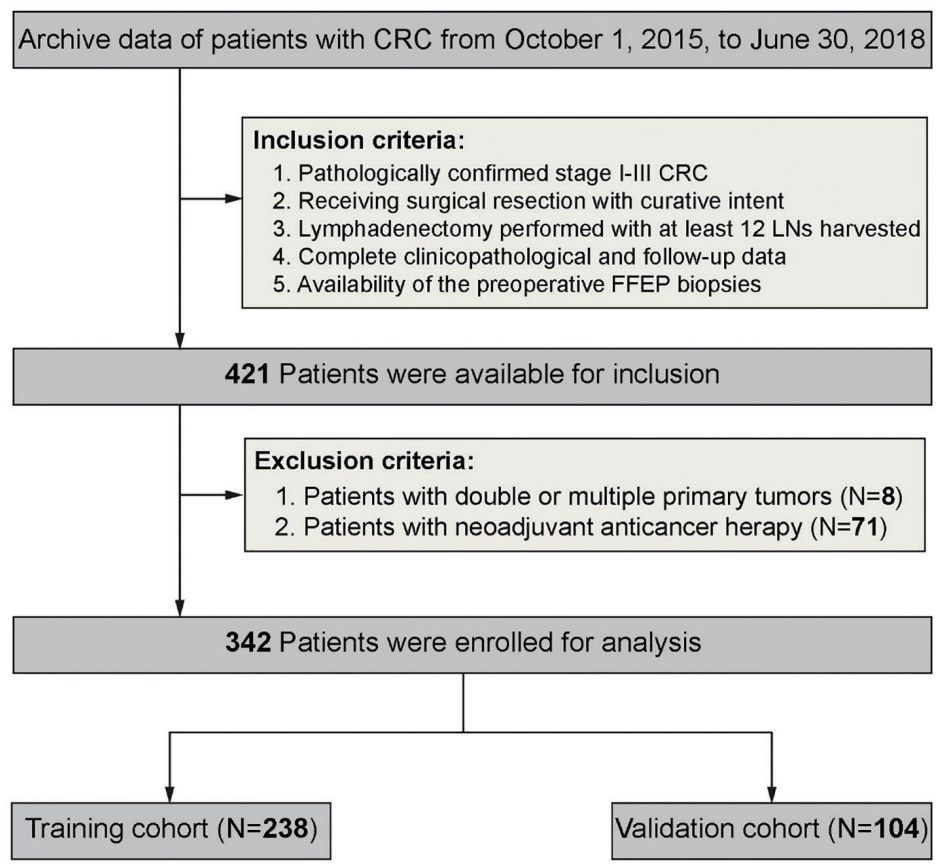

Figure S1 Recruitment scheme for patients in this study. CRC, colorectal cancer; FFEP, formalin-fixed and paraffin-embedded; LN, lymph node.
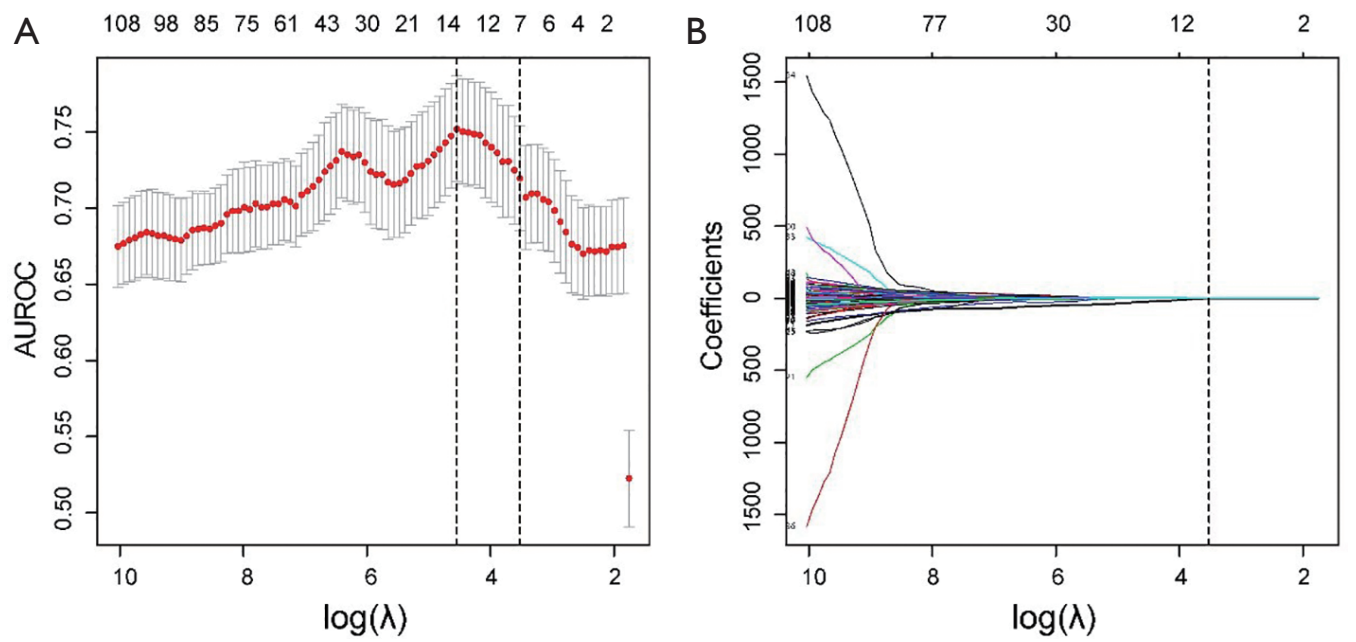

Figure S2 Collagen feature selection using the LASSO regression method. (A) Tuning parameter ( $\lambda$ ) selection in the LASSO regression method using 10-fold cross-validation. Dotted vertical lines were drawn at the optimal values by the minimum criteria (left) and the 1-SE criteria (right). A $\lambda$ value of 0.03 , with $\log (\lambda)=-3.51$, was chosen by 10 -fold cross-validation via 1 -SE criteria. (B) LASSO coefficient profiles of the 148 collagen features. A dotted vertical line was drawn at the value [ $\log (\lambda)=-3.51]$ using 10 -fold cross-validation, wherein the optimal $\lambda$ resulted in 7 nonzero coefficients. SE, standard error; AUROC, area under the receiver operator characteristic curve; LASSO, least absolute shrinkage and selection operator. 

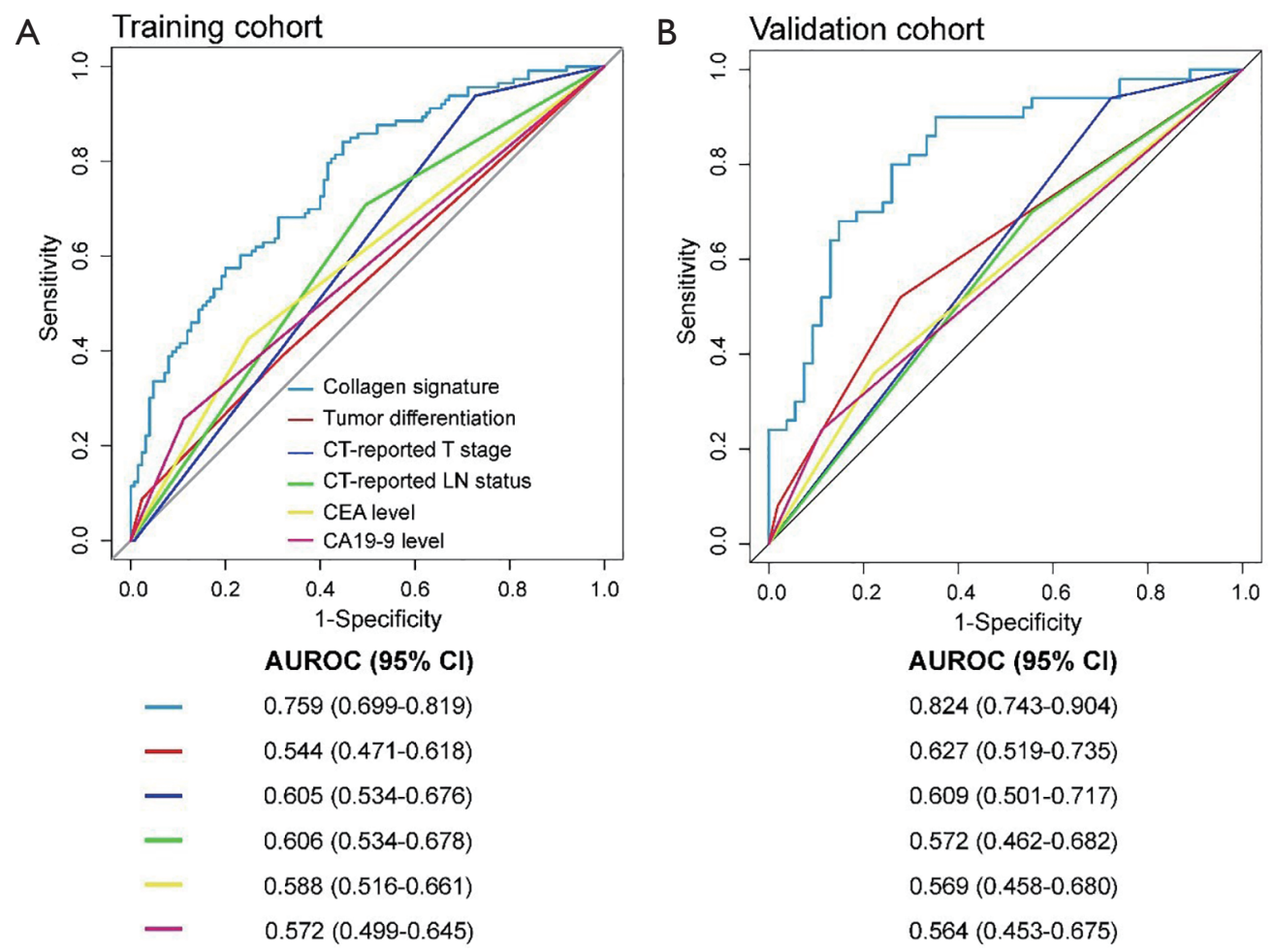

Figure S3 Discrimination of the collagen signature and preoperative risk factors for LN status in the (A) training and (B) validation cohorts. CT, computed tomography; CEA, carcinoembryonic antigen; CA, carbohydrate antigen; LN, lymph node; AUROC, area under the receiver operator characteristic curve; CI, confidence interval.
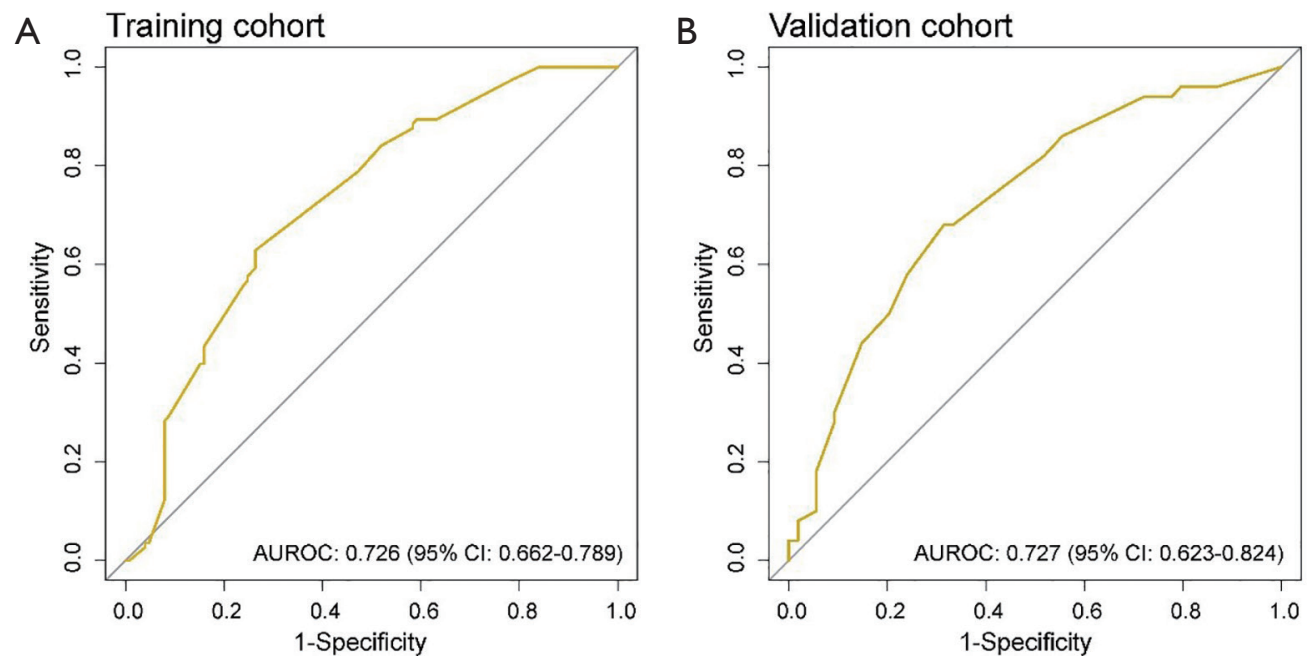

Figure S4 Performance evaluation of the clinicopathological model in the (A) training and (B) validation cohorts. AUROC, area under the receiver operator characteristic curve; CI, confidence interval. 


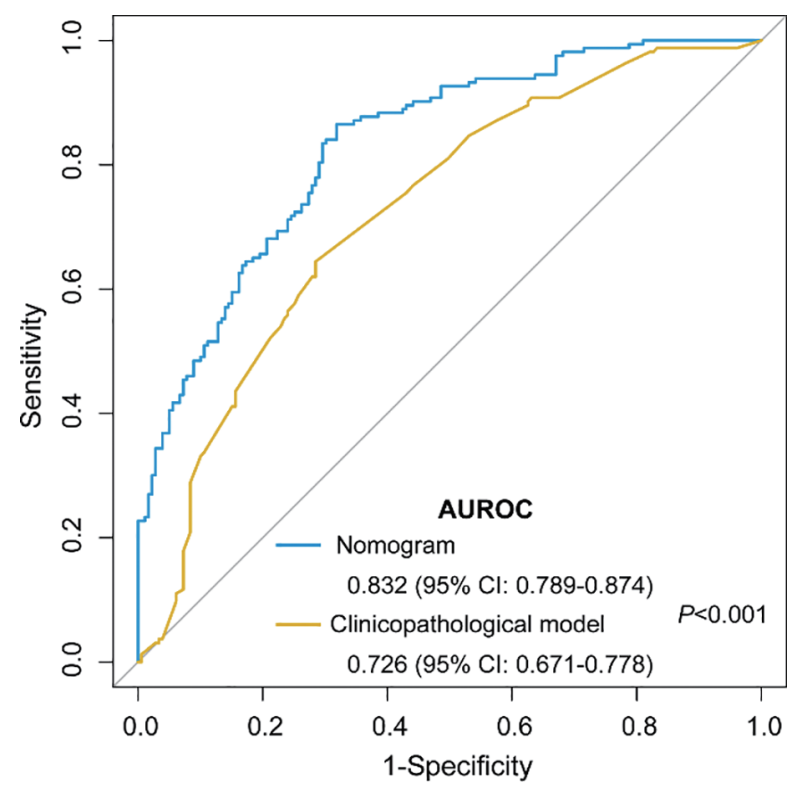

Figure S5 Performance comparison between the collagen signature-based nomogram and the clinicopathological model for all patients. The blue line represents the collagen signature-based nomogram, which consists of preoperative tumor differentiation, CT-reported T stage, CT-reported LN status, CEA level, CA 19-9 level and collagen signature, with an AUROC of 0.832 (95\% CI: 0.789-0.874). The yellow line represents the clinicopathological model, which consists of preoperative tumor differentiation, CT-reported T stage, CTreported LN status, CEA level and CA 19-9 level, with an AUROC of 0.726 (95\% CI: 0.671-0.778). CT, computed tomography; CEA, carcinoembryonic antigen; CA, carbohydrate antigen; LN, lymph node; AUROC, area under the receiver operator characteristic curve; CI, confidence interval.

A
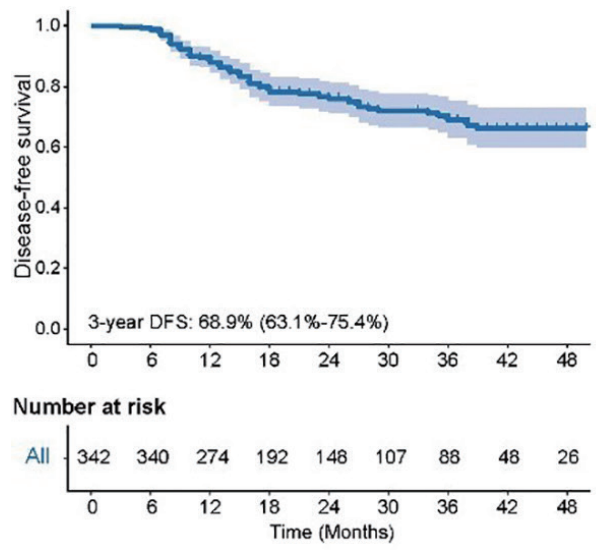

B
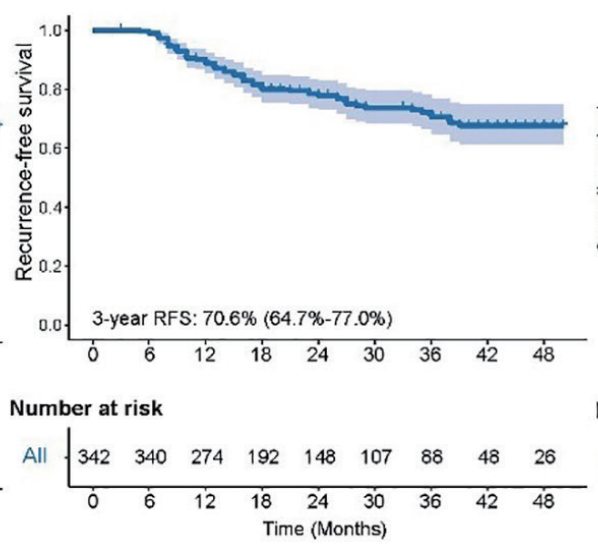

C

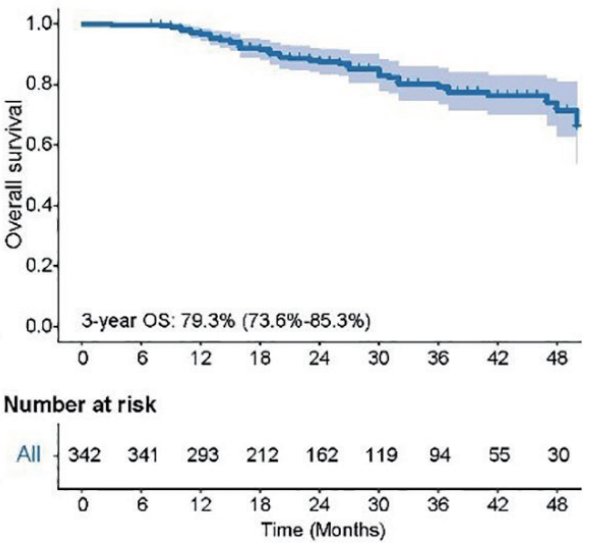

Figure S6 Kaplan-Meier survival analysis of all patients. (A) Disease-free survival of all patients. (B) Recurrence-free survival of all patients. (C) Overall survival of all patients. 


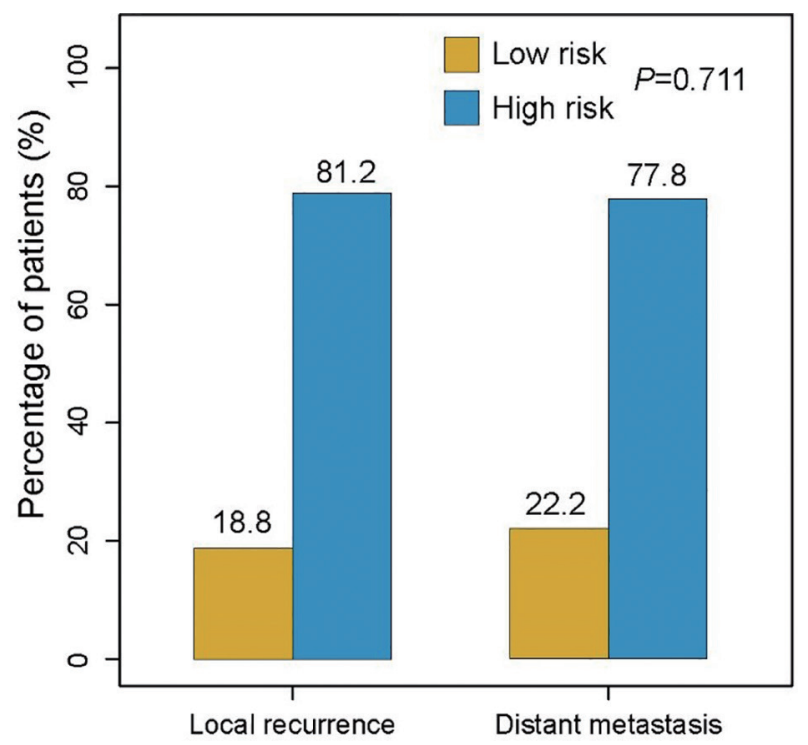

Figure S7 Subgroup analyses of pattern of recurrence in different nomogram-predicted risk groups.

Table S1 List of collagen feature variables

\begin{tabular}{ll}
\hline Number of features & \\
\hline Morphological features & Collagen area (mean and variation) \\
$1-2$ & Collagen number (mean and variation) \\
$3-4$ & Collagen length (mean and variation) \\
$5-6$ & Collagen width (mean and variation) \\
$7-8$ & Collagen straightness (mean and variation) \\
$9-10$ & Collagen cross-link density (mean and variation) \\
$11-12$ & Collagen orientation (mean and variation) \\
$13-14$ & \\
Textural features & Mean; variation; skewness; kurtosis; entropy; energy \\
$15-20$ & Contrast, correlation, energy and homogeneity of the gray-level co-occurrence matrix (GLCM) at five different \\
$21-100$ & pixel distances ( $=1 ; 2 ; 3 ; 4 ; 5)$ with four different directions $\left(\alpha=0^{\circ} ; 45^{\circ} ; 90^{\circ} ; 135^{\circ}\right)$ \\
$101-148$ & Mean and variation of the convolution over the image with the Gabor filter at four scales with six orientations \\
\hline
\end{tabular}


Table S2 Stratified analysis of the association between the collagen signature and LN metastasis

\begin{tabular}{|c|c|c|c|c|}
\hline \multirow{2}{*}{ Variable } & \multicolumn{2}{|c|}{ Collagen signature, median (IQR) } & \multirow{2}{*}{$P$} & \multirow{2}{*}{ AUROC $(95 \% \mathrm{Cl})$} \\
\hline & With LN metastasis & Without LN metastasis & & \\
\hline \multicolumn{5}{|l|}{ Training cohort } \\
\hline \multicolumn{5}{|l|}{ Sex } \\
\hline Male & $0.130(-0.261$ to 0.539$)$ & $-0.325(-0.770$ to 0.053$)$ & $<0.001$ & $0.716(0.627$ to 0.804$)$ \\
\hline Female & $0.146(-0.159$ to 0.498$)$ & $-0.431(-0.854$ to -0.050$)$ & $<0.001$ & $0.814(0.734$ to 0.894$)$ \\
\hline \multicolumn{5}{|l|}{ Tumor location } \\
\hline Rectum & $0.144(-0.222$ to 0.529$)$ & $-0.321(-0.766$ to 0.050$)$ & $<0.001$ & 0.743 (0.637 to 0.848$)$ \\
\hline Colon & $0.131(-0.200$ to 0.498$)$ & $-0.432(-0.796$ to -0.005$)$ & $<0.001$ & 0.769 (0.696 to 0.842 ) \\
\hline \multicolumn{5}{|c|}{ Preoperative tumor differentiation } \\
\hline Well & $0.069(-0.268$ to 0.494$)$ & $-0.267(-0.771$ to 0.009$)$ & $<0.001$ & 0.700 (0.618 to 0.782$)$ \\
\hline $\begin{array}{l}\text { Moderate, poor and } \\
\text { undifferentiated }\end{array}$ & $0.233(-0.056$ to 0.638$)$ & $-0.511(-0.810$ to -0.162$)$ & $<0.001$ & 0.856 (0.776 to 0.936$)$ \\
\hline \multicolumn{5}{|c|}{ Preoperative histological type } \\
\hline Adenocarcinoma & $0.146(-0.194$ to 0.503$)$ & $-0.380(-0.777$ to 0.000$)$ & 0.005 & $0.748(0.596$ to 0.900$)$ \\
\hline Mucinous & $-0.169(-0.708$ to 0.577$)$ & $-0.820(-0.931$ to -0.599$)$ & 0.114 & 0.917 (0.691 to 0.999$)$ \\
\hline \multicolumn{5}{|l|}{ CT-reported tumor size } \\
\hline$\leq 4 \mathrm{~cm}$ & $0.148(-0.170$ to 0.498$)$ & $-0.495(-0.847$ to -0.065$)$ & $<0.001$ & $0.847(0.780$ to 0.914$)$ \\
\hline$>4 \mathrm{~cm}$ & $0.117(-0.288$ to 0.644$)$ & $-0.166(-0.766$ to 0.063$)$ & 0.001 & $0.682(0.586$ to 0.778$)$ \\
\hline \multicolumn{5}{|l|}{ CT-reported T stage } \\
\hline $\mathrm{T} 1$ and $\mathrm{T} 2$ & $0.287(-0.169$ to 0.510$)$ & $-0.506(-1.000$ to -0.019$)$ & 0.008 & 0.824 (0.659 to 0.988$)$ \\
\hline T3 and T4 & $0.118(-0.211$ to 0.501$)$ & $-0.340(-0.732$ to -0.001$)$ & $<0.001$ & 0.745 (0.678 to 0.813$)$ \\
\hline \multicolumn{5}{|l|}{ CT-reported LN status } \\
\hline Negative & $0.267(-0.176$ to 0.695$)$ & $-0.444(-0.775$ to -0.041$)$ & $<0.001$ & 0.788 (0.692 to 0.885$)$ \\
\hline Positive & $0.083(-0.212$ to 0.498$)$ & $-0.314(-0.826$ to 0.009$)$ & 0.001 & $0.740(0.660$ to 0.820$)$ \\
\hline \multicolumn{5}{|l|}{ CEA level } \\
\hline Normal & $0.113(-0.224$ to 0.424$)$ & $-0.302(-0.740$ to 0.033$)$ & $<0.001$ & 0.715 (0.636 to 0.795$)$ \\
\hline Elevated & $0.229(-0.178$ to 0.890$)$ & $-0.524(-0.931$ to -0.153$)$ & $<0.001$ & 0.850 (0.768 to 0.932$)$ \\
\hline \multicolumn{5}{|l|}{ CA 19-9 level } \\
\hline Normal & $0.145(-0.199$ to 0.497$)$ & $-0.382(-0.775$ to -0.001$)$ & $<0.001$ & 0.758 (0.691 to 0.825$)$ \\
\hline Elevated & $0.077(-0.217$ to 0.789$)$ & $-0.487(-1.079$ to -0.113$)$ & 0.002 & 0.791 ( 0.643 to 0.938 ) \\
\hline \multicolumn{5}{|l|}{ Validation cohort } \\
\hline \multicolumn{5}{|l|}{ Sex } \\
\hline Male & $0.282(-0.090$ to 1.180$)$ & $-0.405(-0.765$ to 0.003$)$ & $<0.001$ & 0.815 (0.716 to 0.914$)$ \\
\hline Female & $0.257(-0.043$ to 0.641$)$ & $-0.506(-0.759$ to -0.109$)$ & $<0.001$ & 0.867 (0.741 to 0.992$)$ \\
\hline \multicolumn{5}{|l|}{ Tumor location } \\
\hline Rectum & $0.315(-0.073$ to 1.226$)$ & $-0.399(-0.764$ to 0.084$)$ & $<0.001$ & 0.809 (0.686 to 0.933$)$ \\
\hline Colon & $0.264(-0.060$ to 0.769$)$ & $-0.437(-0.753$ to -0.120$)$ & $<0.001$ & 0.834 (0.720 to 0.947$)$ \\
\hline \multicolumn{5}{|c|}{ Preoperative tumor differentiation } \\
\hline Well & $0.273(-0.196$ to 0.797$)$ & $-0.425(-0.795$ to -0.025$)$ & $<0.001$ & 0.808 (0.694 to 0.922$)$ \\
\hline $\begin{array}{l}\text { Moderate, poor and } \\
\text { undifferentiated }\end{array}$ & $0.302(-0.010$ to 1.132$)$ & $-0.397(-0.685$ to 0.093$)$ & $<0.001$ & 0.823 (0.684 to 0.962$)$ \\
\hline Preoperative histologic & & & & \\
\hline Adenocarcinoma & $0.146(-0.194$ to 0.503$)$ & $-0.425(-0.762$ to -0.013$)$ & $<0.001$ & 0.824 (0.743 to 0.905$)$ \\
\hline Mucinous & - & - & - & - \\
\hline CT-reported tumor size & & & & \\
\hline$\leq 4 \mathrm{~cm}$ & 0.464 (0.012 to 1.234$)$ & $-0.506(-0.749$ to -0.038$)$ & $<0.001$ & 0.867 (0.767 to 0.967$)$ \\
\hline$>4 \mathrm{~cm}$ & $0.210(-0.196$ to 0.717$)$ & $-0.395(-0.772$ to -0.019$)$ & $<0.001$ & 0.781 (0.655 to 0.906$)$ \\
\hline CT-reported T stage & & & & \\
\hline $\mathrm{T} 1$ and $\mathrm{T} 2$ & 0.283 (0.167 to 0.818$)$ & $-0.109(-0.778$ to 0.093$)$ & 0.086 & 0.822 (0.632 to 0.999$)$ \\
\hline T3 and T4 & $0.264(-0.089$ to 1.048$)$ & $-0.425(-0.759$ to -0.181$)$ & $<0.001$ & $0.847(0.764$ to 0.930$)$ \\
\hline CT-reported LN status & & & & \\
\hline Negative & $0.264(-0.055$ to 0.818$)$ & $-0.447(-0.684$ to -0.006$)$ & 0.002 & 0.789 (0.639 to 0.939$)$ \\
\hline Positive & $0.315(-0.089$ to 1.102$)$ & $-0.401(-0.795$ to -0.074$)$ & $<0.001$ & 0.844 (0.748 to 0.940$)$ \\
\hline CEA level & & & & \\
\hline Normal & 0.299 (0.002 to 1.131$)$ & $-0.478(-0.761$ to -0.081$)$ & $<0.001$ & 0.836 (0.741 to 0.930$)$ \\
\hline Elevated & $0.254(-0.112$ to 0.694$)$ & $-0.316(-0.749$ to 0.019$)$ & 0.005 & 0.806 (0.651 to 0.960$)$ \\
\hline CA 19-9 level & & & & \\
\hline Normal & $0.273(-0.037$ to 0.994$)$ & $-0.447(-0.749$ to -0.044$)$ & $<0.001$ & $0.833(0.746$ to 0.921$)$ \\
\hline Elevated & $0.286(-0.154$ to 1.126$)$ & $-0.275(-0.962$ to 0.206$)$ & 0.049 & 0.792 (0.573 to 0.999 ) \\
\hline
\end{tabular}

IQR, interquartile range; CT, computed tomography; CEA, carcinoembryonic antigen; CA, carbohydrate antigen; LN, lymph node; AUROC, area under the receiver operator characteristic curve; $\mathrm{Cl}$, confidence interval. 
Table S3 Multicollinearity assessment of the prediction model

\begin{tabular}{lcc}
\hline \multirow{2}{*}{ Variable } & \multicolumn{2}{c}{ Collinearity statistics } \\
\cline { 2 - 3 } & Tolerance & Variance inflation factor \\
\hline CT-reported T stage & 0.924 & 1.083 \\
CT-reported LN status & 0.949 & 1.053 \\
Preoperative tumor differentiation & 0.989 & 1.011 \\
CEA level & 0.871 & 1.148 \\
CA 19-9 level & 0.867 & 1.154 \\
Collagen signature & 0.951 & 1.052
\end{tabular}

CT, computed tomography; CEA, carcinoembryonic antigen; CA, carbohydrate antigen; LN, lymph node.

Table S4 Univariate and multivariate analyses in the training cohort without the collagen signature

\begin{tabular}{|c|c|c|c|c|}
\hline \multirow{2}{*}{ Variable } & Univariate analysis & \multirow{2}{*}{$\mathrm{P}$} & Multivariate analysis & \multirow{2}{*}{$P$} \\
\hline & OR $(95 \% \mathrm{Cl})$ & & OR $(95 \% \mathrm{Cl})$ & \\
\hline Age & $0.992(0.972-1.013)$ & 0.458 & - & - \\
\hline Sex (female vs. male) & $1.429(0.856-2.385)$ & 0.172 & - & - \\
\hline Location (colon vs. rectum) & $0.889(0.521-1.515)$ & 0.665 & - & - \\
\hline Preoperative histological type (mucinous vs. adenocarcinoma) & $1.492(0.327-6.817)$ & 0.605 & - & - \\
\hline Preoperative tumor differentiation & & 0.046 & & 0.092 \\
\hline Well & Reference & $>0.99$ & Reference & $>0.99$ \\
\hline Moderate & $1.132(0.644-1.989)$ & 0.666 & $1.221(0.662-2.252)$ & 0.523 \\
\hline Poor and undifferentiated & $4.106(1.087-15.506)$ & 0.037 & $4.515(1.151-17.715)$ & 0.031 \\
\hline CT-reported tumor size (>4 vs. $\leq 4 \mathrm{~cm}$ ) & $0.904(0.543-1.504)$ & 0.697 & & - \\
\hline CT-reported T stage (T3 and T4 vs. T1 and T2) & $5.658(2.393-13.375)$ & $<0.001$ & $4.323(1.757-10.634)$ & 0.001 \\
\hline CT-reported LN status (positive vs. negative) & $2.463(1.441-4.210)$ & 0.001 & $2.240(1.252-4.007)$ & 0.007 \\
\hline CEA level (elevated vs. normal) & $2.239(1.290-3.886)$ & 0.004 & $1.687(0.906-3.141)$ & 0.099 \\
\hline CA 19-9 level (elevated vs. normal) & $2.737(1.362-5.501)$ & 0.005 & $2.055(0.940-4.489)$ & 0.071 \\
\hline
\end{tabular}

OR, odd ratio; CT, computed tomography; CEA, carcinoembryonic antigen; CA, carbohydrate antigen; LN, lymph node; $\mathrm{Cl}$, confidence interval.

Table S5 Performance comparison between the nomogram and clinicopathological model

\begin{tabular}{lll}
\hline Models & AUROC $(95 \% \mathrm{Cl})$ & $\mathrm{P}$ \\
\hline Training cohort & & $<0.001$ \\
Nomogram & $0.826(0.774-0.877)$ & 0.004 \\
Clinicopathological model & $0.726(0.662-0.789)$ & \\
Validation cohort & & \\
Nomogram & $0.846(0.773-0.918)$ & $<0.001$ \\
Clinicopathological model & $0.727(0.623-0.824)$ & \\
Total cohort & $0.832(0.789-0.874)$ & $0.726(0.671-0.778)$ \\
Nomogram & & \\
Clinicopathological model &
\end{tabular}

AUROC, area under the receiver operator characteristic curve; $\mathrm{Cl}$, confidence interval. 
Table S6 Univariate and multivariate Cox regression analyses of preoperative predictors for DFS, RFS and OS

\begin{tabular}{|c|c|c|c|c|}
\hline \multirow{2}{*}{ Variable } & Univariate analysis & \multirow{2}{*}{$\mathrm{P}$} & \multirow{2}{*}{$\frac{\text { Multivariate analysis }}{\mathrm{HR}(95 \% \mathrm{Cl})}$} & \multirow{2}{*}{$\mathrm{P}$} \\
\hline & $\mathrm{HR}(95 \% \mathrm{Cl})$ & & & \\
\hline \multicolumn{5}{|l|}{ Disease-free survival } \\
\hline Age & $1.005(0.988-1.023)$ & 0.574 & - & - \\
\hline Sex (female vs. male) & $0.985(0.637-1.523)$ & 0.945 & - & - \\
\hline Location (colon vs. rectum) & $0.887(0.571-1.377)$ & 0.593 & - & - \\
\hline Preoperative histological type (mucinous vs. adenocarcinoma) & $2.951(1.078-8.082)$ & 0.035 & $2.069(0.752-5.695)$ & 0.159 \\
\hline Preoperative tumor differentiation & & 0.174 & & - \\
\hline Well & Reference & $>0.99$ & - & - \\
\hline Moderate & $1.045(0.657-1.661)$ & 0.853 & - & - \\
\hline Poor and undifferentiated & $2.036(0.958-4.329)$ & 0.065 & - & - \\
\hline CT-reported tumor size ( $>4 \mathrm{~cm}$ vs. $\leq 4 \mathrm{~cm})$ & $1.113(0.723-1.712)$ & 0.627 & - & - \\
\hline CT-reported T stage (T3 and T4 vs. T1 and T2) & $3.440(1.392-8.498)$ & 0.007 & $1.755(0.986-3.947)$ & 0.055 \\
\hline CT-reported LN status (positive vs. negative) & $1.376(0.878-2.155)$ & 0.164 & - & - \\
\hline CEA level (elevated vs. normal) & $2.056(1.335-3.167)$ & 0.001 & $1.449(0.914-2.300)$ & 0.115 \\
\hline CA 19-9 level (elevated vs. normal) & $2.354(1.463-3.787)$ & $<0.001$ & $1.563(0.941-2.594)$ & 0.084 \\
\hline \multicolumn{5}{|l|}{ Recurrence-free survival } \\
\hline Age & $1.001(0.983-1.019)$ & 0.943 & - & - \\
\hline Sex (female vs. male) & $0.988(0.629-1.552)$ & 0.960 & - & - \\
\hline Location (colon vs. rectum) & $0.883(0.609-1.533)$ & 0.883 & - & - \\
\hline Preoperative histological type (mucinous vs. adenocarcinoma) & $3.226(1.175-8.855)$ & 0.023 & $2.232(0.810-6.154)$ & 0.121 \\
\hline Preoperative tumor differentiation & & 0.264 & & - \\
\hline Well & Reference & $>0.99$ & - & - \\
\hline Moderate & $1.105(0.685-1.781)$ & 0.682 & - & - \\
\hline Poor and undifferentiated & $1.953(0.874-4.364)$ & 0.103 & - & - \\
\hline CT-reported tumor size (>4 cm vs. $\leq 4 \mathrm{~cm}$ ) & $1.066(0.682-1.668)$ & 0.778 & - & - \\
\hline CT-reported T stage (T3 and T4 vs. T1 and T2) & $4.038(1.476-11.049)$ & 0.007 & $2.005(0.685-5.872)$ & 0.205 \\
\hline CT-reported LN status (positive vs. negative) & $1.368(0.859-2.179)$ & 0.187 & - & - \\
\hline CEA level (elevated vs. normal) & $2.254(1.441-3.525)$ & $<0.001$ & $1.570(0.974-2.531)$ & 0.064 \\
\hline CA 19-9 level (elevated vs. normal) & $2.484(1.523-4.051)$ & $<0.001$ & $1.589(0.944-2.675)$ & 0.081 \\
\hline Nomogram-predicted risk (high vs. low) & $3.567(2.050-6.207)$ & $<0.001$ & $2.404(1.308-4.418)$ & 0.005 \\
\hline \multicolumn{5}{|l|}{ Overall survival } \\
\hline Age & $1.001(0.979-1.024)$ & 0.935 & - & - \\
\hline Sex (female vs. male) & $0.902(0.512-1.589)$ & 0.721 & - & - \\
\hline Location (colon vs. rectum) & $0.777(0.443-1.363)$ & 0.379 & - & - \\
\hline Preoperative histological type (mucinous vs. adenocarcinoma) & $4.610(1.654-12.851)$ & 0.003 & $2.018(0.477-8.538)$ & 0.340 \\
\hline Preoperative tumor differentiation & & 0.037 & & 0.786 \\
\hline Well & Reference & $>0.99$ & Reference & $>0.99$ \\
\hline Moderate & $1.115(0.609-2.041)$ & 0.725 & $1.005(0.548-1.842)$ & 0.988 \\
\hline Poor and undifferentiated & $2.983(1.269-7.012)$ & 0.012 & $1.503(0.460-4.913)$ & 0.500 \\
\hline CT-reported tumor size (>4 vs. $\leq 4 \mathrm{~cm}$ ) & $1.482(0.844-2.600)$ & 0.171 & - & - \\
\hline CT-reported T stage (T3 and T4 vs. T1 and T2) & $2.528(0.910-7.025)$ & 0.075 & - & - \\
\hline CT-reported LN status (positive vs. negative) & $1.599(0.890-2.876)$ & 0.117 & - & - \\
\hline CEA level (elevated vs. normal) & $2.098(1.204-3.654)$ & 0.009 & $1.397(0.773-2.526)$ & 0.268 \\
\hline (C) Annals ${ }^{\prime}$ f Translational Medicine, All rights reserved. & $2.975(1.654-5.350)$ & $<0.001^{h}$ & 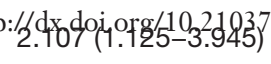 & $\mathrm{n}_{\overline{0}}-28 \overline{2} \overline{0} 0^{565}$ \\
\hline Nomogram-predicted risk (high vs. low) & $3.763(1.876-7.545)$ & $<0.001$ & $2.774(1.319-5.831)$ & 0.007 \\
\hline
\end{tabular}

$\mathrm{CT}$, computed tomography; CEA, carcinoembryonic antigen; CA, carbohydrate antigen; LN, lymph node; HR, hazard ratio; Cl, confidence interval; DFS, disease-free survival; OS, overall survival. 